\title{
L’impact redistributif des impôts indirects en France
}

\author{
Gérard Forgeot* (18 Bd Adolphe Pinard, 75675 Paris Cedex 14 \\ gerard.forgeot@insee.fr) \\ INSEE
}

\author{
Christophe Starzec*(18 Bd Adolphe Pinard, 75675 Paris Cedex 14 starzec@univ- \\ paris1.fr) \\ TEAM-CNRS, INSEE
}

\section{Codes JEL: H24, D31, D12}

Mots Clés: Impôts indirects, redistribution, taux d'imposition

Key words : Indirect taxes, redistribution, tax rates

\begin{abstract}
Résumé
La structure, le poids et les effets redistributifs de la fiscalité indirecte en France sont mesurés en utilisant une nouvelle base de données construite à partir de l'Enquête Budget de famille 2001 de l'Insee. Pour chaque poste de dépense, les montants des différentes composantes de la fiscalité indirecte ont été calculés grâce notamment au traitement de l'information sur les quantités consommées. Il est dès lors possible d'obtenir des taux et des montants moyens d'imposition au titre des différentes taxes sur la consommation (TVA, TIPP, droits d'accises...). Les effets redistributifs de ces taxes varient sensiblement selon les caractéristiques des ménages, notamment leur revenu. L'effet globalement anti-redistributif de la fiscalité indirecte contribue à augmenter de 1,3 point le coefficient de Gini. Une analyse économétrique du montant des taxes payées en fonction du revenu et des caractéristiques socio-démographiques témoigne d'un effet prépondérant du revenu, avec une élasticité revenu de l'ensemble de la fiscalité indirecte positive, mais inférieure à l'unité $(0,4)$. Toutefois cet effet revenu varie sensiblement selon la nature de la taxe indirecte considérée. Ainsi, l'élasticité au revenu des taxes sur le tabac est proche de zéro, tandis que celle de la TIPP est d'environ 0,3. Une simulation des effets des réformes successives de la TVA observées depuis 1976 a été appliquée à la structure des ménages donnée par l'enquête Budget 2001. Elle met en évidence une diminution du taux de prélèvement de 1,5 point sur l'ensemble des ménages, mais sans effet sur les disparités de taux d'effort entre les ménages aisés et modestes.
\end{abstract}

\section{Summary}

The importance and redistributive effects of indirect taxation in France are measured using an original data basis derived from Household Budget Survey 2001. The detailed disaggregated information on the indirect tax rates was computed for each spending. The obtained medium tax rates for the total indirect taxation and all its components show the global antiredistributive effects leading to a large increase in inequalities (the Gini coefficient increases by 1.3 point). An econometric analysis of main determinants influencing amounts of paid taxes confirm the crucial role of income with an elasticity positive, and inferior to one (0.4). However, this income effect varies strongly with respect to the specific considered tax tobacco tax income elasticity is close to zero and those for petrol around 0.3.

The simulation of VAT rates evolution since 1976 (principal component of indirect taxes) is applied to the population and consumption structure of 2001. Reducing VAT tax rate over this period leads to a 1.5 point decrease in the tax rate for the whole population. However, the 
disparities in terms of tax rates between poor and rich households remain practically unchanged over the period.

\section{INTRODUCTION}

La fiscalité indirecte représente, en France, la première recette budgétaire de l'État. Or, ce sont les impôts les moins étudiés en regard à d'autres prélèvements. Les effets redistributifs de ces taxes, ainsi que l'impact des changements de taux sur les comportements individuels sont peu connus. Seules quelques études antérieures, dans un passé relativement lointain, ont apporté un éclairage généralement restreint sur cette question. Le travail le plus récent de Trannoy et al (2003) a rappelé la complexité de la législation en la matière et, corrélativement, la difficulté d'en modéliser l'impact.

Une étude sur l'évolution de la redistributivité du système socio fiscal (Murat, Roth, Starzec, 2001) a mis en relief le rôle de l'évolution de la TVA comparé à l'ensemble des prélèvements (modèle INES de l'INSEE). Des contributions plus anciennes ont porté sur l'évaluation des réformes réelles et potentielles de la fiscalité indirecte sur la répartition des revenus sans prendre en compte les éventuels changements de comportements en matière de consommation des ménages induits par ces réformes (David, Lhommeau, Starzec 1999), ou avec une modélisation de ces comportements (Gardes, Lhommeau, Starzec 1998, Nichèle, Robin 1993, Baccouche, Laisney, 1986 et 1990). D’une manière générale, ces études ont montré un effet global faible de la fiscalité indirecte sur la répartition des revenus, y compris lors de simulations de réformes d'assez grande ampleur. Dans ces études, seule la TVA a été traitée d'une manière exhaustive, les autres taxes indirectes ayant souvent fait l'objet d'un traitement extrêmement simplifié en raison, notamment, de l'absence de données à un niveau suffisamment désagrégé pour permettre de les prendre en compte de façon satisfaisante.

Cet article reprend la problématique des effets redistributifs de la fiscalité indirecte, en prenant en compte la quasi totalité des taxes indirectes. Il s'appuie pour cela sur les données de l'enquête Budget de famille de l'Insee réalisée en 2000-2001, qui innove par rapport aux éditions antérieures : outre le détail des dépenses, les quantités correspondantes sont aussi collectées. Partant de la nomenclature des dépenses, la fiscalité indirecte (TVA, droits d'accises, TIPP et autres prélèvements indirects) est calculée sur chaque poste de consommation pour chacun des ménages. Les définitions des différentes taxes indirectes en France, ainsi que leur poids dans l'ensemble des prélèvements, sont présentés dans la section 1. 
La section 2 présente la base de données utilisée, ainsi que le poids de la fiscalité indirecte selon différentes caractéristiques socio-démographiques des ménages. L'impact redistributif de ces taxes, et leurs effets sur les inégalités, sont aussi analysés. En évolution temporelle, l'analyse est centrée sur la seule TVA. Les conséquences des réformes successives engagées de puis 1976 sont ainsi présentées.

Une analyse économétrique des déterminants du montant des impôts indirects payés par les ménages en fonction de leurs caractéristiques est proposée dans la section 3. Sont estimées en particulier les élasticités revenu pour l'ensemble des taxes indirectes, ainsi que pour les principales taxes spécifiques (TIPP, tabac, alcool...).

\section{1-La fiscalité indirecte en France ${ }^{1}$}

\subsection{Un poids important de la fiscalité sur la consommation en France}

En 2001, les prélèvements obligatoires représentent 659 milliards d'euros. Les ménages ont contribué à hauteur de 47,6 \% à ce montant. Dans le budget de l'État (hors administrations publiques locales et prélèvements sociaux), la fiscalité indirecte représente près de $42 \% \mathrm{du}$ total, dont 30,7\% pour la Taxe sur la valeur ajoutée (TVA) (tableau 1). Le taux des prélèvements obligatoires a sensiblement augmenté au cours des vingt dernières années : la part des cotisations sociales et de la fiscalité directe dans le PIB se sont accrues, tandis que celle de la fiscalité indirecte a légèrement diminué, tout en conservant sa position de principale recette budgétaire.

Parmi les impôts à la consommation, on peut distinguer la TVA, les droits d'accises et les autres taxes indirectes.

\footnotetext{
${ }^{1}$ Ces informations sur la fiscalité indirecte ont été en grande partie collectées par Agnès AMIEL, dans le cadre de son stage de DESS « Méthodes quantitatives et modélisation pour l'entreprise », effectué à l'INSEE en 2003.
} 
Tableau 1 : Les prélèvements obligatoires au titre du budget de l’État en 2001

\begin{tabular}{|c|c|c|}
\hline & en millions d' euros & part du total en $\%$ \\
\hline $\begin{array}{l}\text { FISCALITE DIRECTE } \\
\text { dont : }\end{array}$ & 112061 & 32,8 \\
\hline Impôt sur le revenu (IR) & 47916 & 14,0 \\
\hline Impôt sur les sociétés & 38962 & 11,4 \\
\hline Impôt sur les revenus de capitaux mobiliers (IRCM) & 2069 & 0,6 \\
\hline Taxe sur les salaires $(\mathrm{TS})$ & 8018 & 2,3 \\
\hline Précompte mobilier & 1691 & 0,5 \\
\hline Impôt de solidarité sur la fortune (ISF) & 2645 & 0,8 \\
\hline Autres & 10760 & 3,2 \\
\hline $\begin{array}{l}\text { FISCALITE INDIRECTE } \\
\text { dont : }\end{array}$ & 142854 & 41,8 \\
\hline TVA & 105041 & 30,7 \\
\hline Taxe intérieure sur les produits pétroliers & 22844 & 6,7 \\
\hline Partie de la Taxe spéciale sur les conventions d'assurance (TCA) & 3353 & 1,0 \\
\hline Droits d'importation & 1404 & 0,4 \\
\hline Autres & 10212 & 3,0 \\
\hline $\begin{array}{l}\text { RECETTES PREAFFECTEES } \\
\text { dont : }\end{array}$ & 80429 & 23,5 \\
\hline CSG globale & 63570 & 18,6 \\
\hline Taxe alcools CNAM & 376 & 0,1 \\
\hline Droits tabacs pour $\mathrm{CMU}$ & 213 & 0,1 \\
\hline Partie des droits sur les alcools et boissons (FOREC) & 2614 & 0,8 \\
\hline Partie des droits sur les tabacs (FOREC) & 7912 & 2,3 \\
\hline Autres & 5744 & 1,7 \\
\hline $\begin{array}{l}\text { RECETTES NON FISCALES } \\
\text { dont : }\end{array}$ & 6694 & 1,9 \\
\hline Produits des jeux exploités par la Française des jeux & 1188 & 0,3 \\
\hline $\begin{array}{l}\text { Prélèvements sur produit des jeux dans les casinos régis loi } \\
15 / 06 / 1907\end{array}$ & 833 & 0,2 \\
\hline Prélèvement sur le pari mutuel & 310 & 0,1 \\
\hline Autres & 4363 & 1,3 \\
\hline TOTAL (en Md€) & 342038 & 100 \\
\hline
\end{tabular}

Source : Ministère de l'Economie, des Finances et de l'industrie (http : //www.finances.gouv.fr/budget)

Au sein de l'OCDE, la France se singularise par la structure de ses prélèvements. En effet, la part de l'impôt sur le revenu est parmi la plus faible au sein des pays membres de l'organisation. A l'opposé, les cotisations de sécurité sociale et la fiscalité indirecte sont les plus élevées, bien au-delà de la moyenne OCDE (graphique 1). 


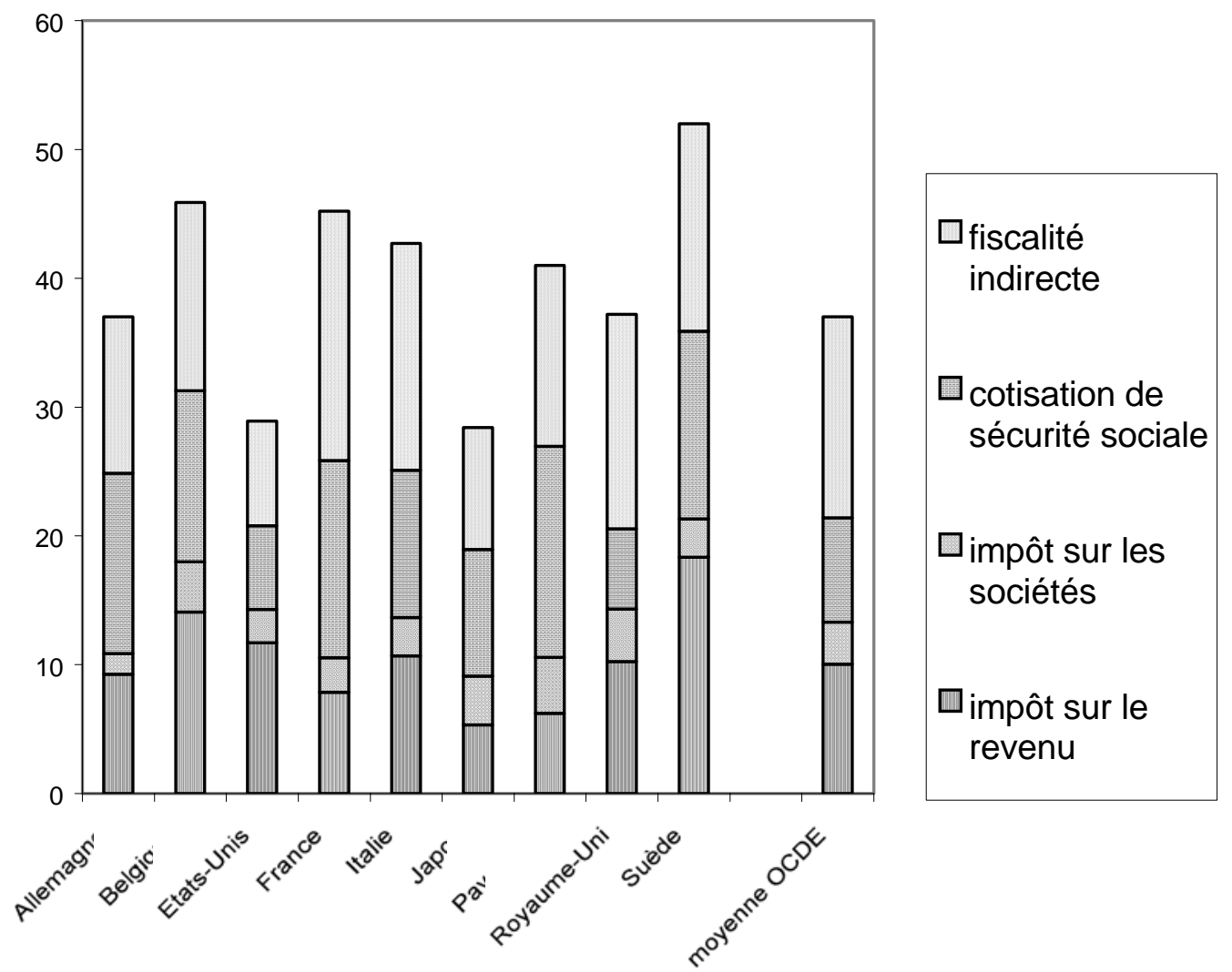

Source : Statistiques des recettes publiques 1965-1998, OCDE, paris, 1999

\subsection{La taxe sur la valeur ajoutée}

La taxe à la valeur ajoutée (TVA), dont le promoteur est Maurice Lauré, a été instituée par une loi du 10 avril 1954, et généralisée à partir du $1^{\mathrm{er}}$ avril 1968. Ce prélèvement a été adopté au niveau européen par la $6^{\text {ème }}$ directive TVA du 17 mai 1977 qui poussait à une harmonisation des législations nationales. La TVA est une taxe ad valorem, c'est-à-dire une taxe proportionnelle à la valeur du bien. Elle repose sur l'activité économique et la consommation et touche en principe l'ensemble des livraisons de biens et prestations de services entre opérateurs économiques.

La TVA est calculée à partir du chiffre d'affaires. La détermination de son montant s'opère à partir d'une "déduction taxe sur taxe». A chaque stade de production et de commercialisation, le redevable est autorisé à déduire au montant de la TVA due la taxe ajoutée au stade précédent, qui figure sur les factures de ses fournisseurs. Il ne verse au fisc que la différence, à savoir la taxe correspondant à sa propre valeur ajoutée. 
La TVA se calcule sur la base du prix de vente hors TVA; le lien entre ces deux prix s'écrit simplement :

$$
\text { prix TVA incluse }=(1+t) \mathrm{x}(\text { prix hors TVA })
$$

où $t$ désigne le taux de TVA applicable au bien ou service considéré (taux normal ou réduit).

\subsubsection{Les taux de TVA}

Le taux de TVA est un paramètre propre à chaque transaction taxable. Deux principaux taux de TVA sont actuellement applicables en France métropolitaine depuis 1 e $1^{\mathrm{er}}$ avril 2000 : le taux normal de $19,6 \%$, le taux réduit de 5,5\% (sans compter les taux spécifiques à la Corse et aux Départements d'Outre-Mer). Un taux spécifique (dit super-réduit) de 2,1\% s'applique aux publications de la presse, à la redevance audiovisuelle, et aux médicaments remboursables par la Sécurité sociale.

\section{Encadré 1. Les taux de TVA applicables en France depuis le $1^{\text {er }}$ avril 2000}

\section{Le taux normal - $\mathbf{1 9 . 6 \%}$}

\section{Le taux réduit $-5.5 \%$ :}

Il s'applique à différentes catégories d'opérations limitativement énumérées (article 278 bis du Code Général des Impôts), notamment (liste non exhaustive) :

* certains biens et services de consommation courante (l'eau, l'essentiel des produits alimentaires, les transports de voyageurs, les médicaments non remboursables par la Sécurité Sociale),

* certains produits culturels (livres, entrées dans les spectacles, entrées dans les musées) ;

* la fourniture de logement en hôtel ou en meublé ;

*les produits de l'horticulture et de la sylviculture depuis 1997

* depuis 1999 , le taux réduit s'applique sur les travaux réalisés par des professionnels portant sur les locaux à usage d'habitation achevés depuis plus de deux ans. Les travaux doivent porter sur la rénovation uniquement sans augmentation de la surface habitable. Le taux réduit s'applique aussi bien aux prestations de main-d'œuvre, aux matières premières et aux équipements, à condition qu'ils soient facturés par les professionnels qui réalisent ces travaux. Toutes les autres catégories de produits sont soumises au taux normal. Cependant, certains biens et services ne sont pas soumis à la TVA tels que les loyers, les services de la poste, les 
services relatifs à l'enseignement, les jeux de hasard ainsi que les assurances (dont le régime de taxation particulier sera vu plus loin).

\section{Un taux spécifique (super réduit) - 2,1\%}

Il s'applique principalement aux publications de presse, à la redevance audiovisuelle et aux médicaments remboursables par la Sécurité sociale.

\subsubsection{Evolution des taux de TVA}

Les taux de TVA ont sensiblement évolué au cours des 30 dernières années ; ce choix répond aux objectifs de politique économique de relancer la consommation en période de crise économique, de collecter plus de recettes comme en 1995, ou de s'harmoniser au niveau européen (Annexe 1).

Tableau 2. L'évolution des taux de TVA (en \%)

\begin{tabular}{|c|c|c|c|c|c|c|}
\hline $\begin{array}{r}\mathrm{Du} \\
\mathrm{Au} \\
\end{array}$ & $\begin{array}{l}01 / 01 / 1968 \\
30 / 11 / 1968 \\
\end{array}$ & $\begin{array}{l}01 / 12 / 1968 \\
31 / 12 / 1969 \\
\end{array}$ & $\begin{array}{l}01 / 01 / 1970 \\
31 / 12 / 1972 \\
\end{array}$ & $\begin{array}{l}1973 \\
1976 \\
\end{array}$ & $\begin{array}{c}1977 \\
30 / 06 / 1982 \\
\end{array}$ & $\begin{array}{l}01 / 07 / 1982 \\
30 / 12 / 1988 \\
\end{array}$ \\
\hline Taux Super Réduit & $/ / /$ & $/ / /$ & /// & $/ / /$ & /// & 5,50 \\
\hline Taux Réduit & 6,38 & 7,53 & 7,50 & 7 & 7 & 7 \\
\hline Taux Normal & 20 & 23,46 & 23 & 20 & 17,60 & 18,60 \\
\hline Taux intermédiaire & 14,92 & 15,65 & 17,60 & 17,60 & 17,60 & $/ / 1$ \\
\hline Taux majoré & 25 & 33,33 & 33,33 & 33,33 & 33,33 & 33,33 \\
\hline
\end{tabular}

\begin{tabular}{|l|r|r|r|r|r|r|}
\hline \multicolumn{1}{|c|}{$\begin{array}{c}\mathrm{Du} \\
\mathrm{Au}\end{array}$} & $01 / 01 / 1989$ & $15 / 09 / 1989$ & $01 / 01 / 1991$ & $01 / 01 / 1993$ & $01 / 08 / 1995$ & $01 / 04 / 2000$ \\
à nos jours
\end{tabular}

Source : Syndicat National Unifié des Impôts (www.snui.fr)

\subsection{Les droits d'accises}

\subsubsection{La fiscalité sur les boissons alcoolisées}

Ce sont des droits indirects de consommation qui frappent certaines catégories de produits. La taxe n'est plus uniquement proportionnelle à la valeur du bien, elle est aussi additive, et exprimée par unité de bien. Une partie de la taxe porte par conséquent sur la quantité et l'autre sur le montant.

Trois catégories de boissons alcoolisées sont distinguées pour les droits à accises (Code Général des Impôts, annexe 2) : les boissons fermentées (les vins, les boissons fermentées autres que le vin, et la bière), les produits intermédiaires et les alcools. Le droit de 
consommation perçu sur les produits intermédiaires diffère selon qu'il s'agit des vins doux naturels ou des autres produits. Les vins sont aussi soumis à un droit de circulation et les bières sont, quant à elles, soumises à des droits spécifiques.

Outre le droit de consommation, les alcools supportent une taxe appelée cotisation CNAM (Caisse Nationale d'Assurance Maladie) et une taxe sur les mélanges de boissons alcooliques et non alcooliques appelée taxe «Premix». Enfin, toutes les boissons alcoolisées sont soumises à la TVA.

Tableau 3. Montant des taxes appliquées aux boissons alcoolisées

\begin{tabular}{|c|c|}
\hline \multicolumn{2}{|l|}{ Alcools } \\
\hline $\begin{array}{l}\text { Droit de consommation } \\
\text { Cotisation Caisse Nationale Assurance Maladie } \\
\text { Taxe premix }\end{array}$ & $\begin{array}{l}1450 € / \text { hectolitre d'alcool pur } \\
130 € / \text { hectolitre de produit } \\
5550 € / \text { hectolitre d'alcool pur }\end{array}$ \\
\hline \multicolumn{2}{|l|}{ Produits intermédiaires } \\
\hline \multicolumn{2}{|c|}{ Droit de consommation sur les produits intermédiaires : } \\
\hline $\begin{array}{l}\text { Vins Doux Naturels } \\
\text { Autres produits }\end{array}$ & $\begin{array}{l}54 € / \text { hectolitre de produit } \\
214 € / \text { hectolitre de produit }\end{array}$ \\
\hline \multicolumn{2}{|l|}{ Boissons fermentées } \\
\hline \multicolumn{2}{|l|}{ Droit de circulation sur les vins : } \\
\hline $\begin{array}{l}\text { Vins mousseux } \\
\text { Autres vins } \\
\text { Cidres, poirés, hydromels et pétillants de raisin }\end{array}$ & $\begin{array}{l}8,40 € / \text { hectolitre de produit } \\
3,40 € / \text { hectolitre de produit } \\
1,20 € / \text { hectolitre de produit }\end{array}$ \\
\hline \multicolumn{2}{|l|}{ Droits spécifiques sur les bières } \\
\hline $\begin{array}{l}\text { Bières dont le degré alcoométrique ne dépasse pas } \\
2,8 \% \text { vol } \\
\text { Autres bières }\end{array}$ & $\begin{array}{l}1,30 € / \text { hectolitre et par degré } \\
\text { alcoométrique } \\
2,60 € / \text { hectolitre et par degré } \\
\text { alcoométrique }\end{array}$ \\
\hline
\end{tabular}

Source : http : // www. douane.gouv.fr

Par exemple, pour une bouteille de whisky de 1 litre titrant $40 \%$ en volume d'alcool, le droit de consommation porte sur le volume d'alcool pur contenu soit $40 \%$ du litre. La taxe collectée sur cette bouteille s'élève à $7,1 €$;

$(1450 \times 0,01 \times 0,40)+(130 \times 0,01)=7,1$

Une taxe sur les boissons mélangées s'applique aux Premix. En guise de rappel, le Premix est un mélange de soda sucré et d'alcool fort titrant de 5 à $8 \%$ en volume d'alcool vendu sous forme de boîtes de 25 à $35 \mathrm{cl}$. Soit une canette de «whisky cola » de $25 \mathrm{cl}$ contenant 6\% d'alcool. Comme les alcools, les Premix sont taxés par hectolitre d'alcool pur. La taxe acquittée sur cette canette s'élève à $0,83 €$ : 
$(5550 \times 0,00025 \times 0,06)=0,825$

Pour les bières, la taxation s'opère par hectolitre de produit et par titre alcoométrique. Ainsi, pour une bière de $33 \mathrm{cl}$ à $4^{\circ}, 0,034 €$ de taxes seront acquittés sur cette bière : $(2,60 \times 0,0033 \times 4)=0,034$.

\subsubsection{La fiscalité sur les tabacs manufacturés}

Les tabacs manufacturés supportent un droit de consommation proportionnel au prix de vente au détail sauf pour les cigarettes. Pour ce produit, le droit de consommation est divisé en deux parties. Une part spécifique porte sur les quantités $(0,1482 €$ pour 20 cigarettes), l'autre part est proportionnelle au prix de vente hors taxe sur un paquet $(55,19 \%$ du prix de vente hors taxe). En outre, l'État exige un minimum de perception de 2,12€ pour 20 cigarettes. Le montant de la taxation pour un paquet de cigarettes est donc constitué :

- $\quad$ soit du minimum de perception

- $\quad$ soit de $55,19 \%$ du prix de vente au détail hors taxe d'un paquet $+0,1482 €$

\section{Graphique 2. Exemple pour un paquet de 20 cigarettes}

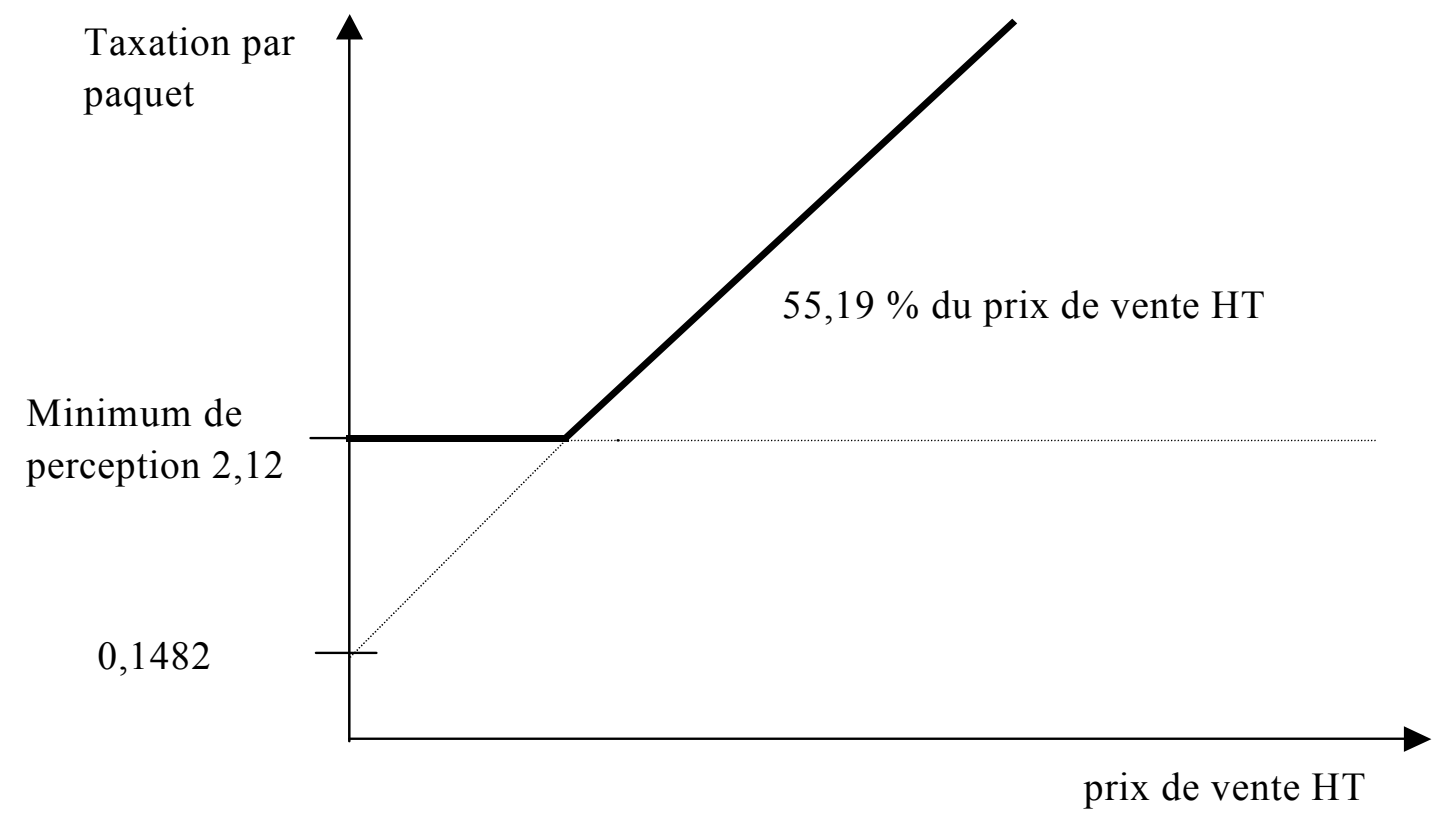

Source : Code Général des Impôts (article 575)

Un mode de taxation analogue s'applique à la plupart des autres tabacs manufacturés. 
Tableau 4. Taxation des tabacs manufacturés hors cigarettes

\begin{tabular}{|l|c|l|}
\hline Groupe de produits & Taux applicable & Minimum de perception en $€$ \\
\hline Cigares & $20 \%$ & $55 €$ pour 1 000 unités \\
\hline $\begin{array}{l}\text { Tabacs fine coupe destinés à rouler } \\
\text { les cigarettes }\end{array}$ & $51,69 \%$ & $56 €$ par kg \\
\hline Autres tabacs à fumer & $47,43 \%$ & $45 €$ par kg \\
\hline Tabacs à priser & $40,89 \%$ & pas de minimum \\
\hline Tabacs à mâcher & $28,16 \%$ & pas de minimum \\
\hline
\end{tabular}

Source : Code Général des Impôts (art 575)

Pour une cartouche de cigarettes d'un montant de $45 €$ hors taxes, le droit de consommation s'élève à $26,32 €$ :

$(45 \times 0,5519)+(10 \times 0,1482)=26,32$

Le droit de consommation porte à la fois sur le nombre de paquets achetés $(0,1482 \times 10)$ et sur le prix de vente $(45 \times 55,19 \%)$.

\subsubsection{La taxe intérieure sur les produits pétroliers}

La Taxe intérieure sur les produits pétroliers (TIPP) est un droit indirect qui porte sur la consommation des carburants sur le territoire national. La taxe est payée par l'acheteur au distributeur de carburants qui la reverse à l'État.

La TIPP est différente selon la nature physique des produits (essence, gazole, fuel domestique). Elle est calculée selon des tarifs prévus par la législation douanière et perçue par la DGDDI (Direction Générale des Douanes et des Droits indirects) lors de la mise en consommation des produits pétroliers sur le marché intérieur. A titre d'exemple, 0,5892 euros de TIPP sont perçues sur un litre de supercarburant sans plomb.

Tableau 5. Les droits acquittés selon les types de carburant

\begin{tabular}{|l|l|r|}
\hline \multicolumn{1}{|c|}{ Désignation des produits } & Unité de perception & Quotité \\
\hline Supercarburant sans plomb & hectolitre & $58,92 €$ \\
\hline Supercarburant sans plomb avec additif & hectolitre & $63,93 €$ \\
\hline Gazole & hectolitre & $38,90 €$ \\
\hline Fioul domestique & hectolitre & $7,88 €$ \\
\hline GPL & 100 kg net & $3,94 €$ \\
\hline
\end{tabular}

Source : Code des douanes (art 265) 
La TIPP a été légèrement aménagée entre 2001 et 2002 avec l'introduction de la TIPP flottante. En effet, durant l'année 2000, les cours du baril de pétrole ont augmenté fortement, et par conséquent, le prix de l'essence à la pompe, dans des proportions telles que les usagers de la route ont protesté. La loi de finances pour 2001 a institué un mécanisme de modulation des tarifs de la TIPP applicable notamment au supercarburant sans plomb, au gazole et au fioul domestique qui a pour but de compenser l'augmentation des recettes de TVA enregistrée du fait de la hausse des prix du brut.

\section{Encadré 2. Article 12 de la loi de finances pour 2001}

D'après l'article 12 de la loi de finances pour 2001 :

« Lorsque le cours moyen du pétrole dénommé « brent daté » varie de plus de $10 \%$ dans les conditions précisées au deuxième alinéa, les tarifs prévus pour les supercarburants, le gazole et le fioul domestique sont corrigés d'un montant égal au produit de la variation en valeur absolue de la moyenne des prix hors taxe de ces produits pétroliers et du taux de $16,388 \%$. Cette correction est effectuée à la baisse en cas de hausse des prix hors taxe et à la hausse dans le cas contraire. »

Lorsque le cours moyen du pétrole brut varie de plus de $10 \%$, les tarifs de la TIPP sont corrigés d'un montant égal au surplus ou à la perte de recettes de TVA :

prix final $=$ prix initial $-16,388 \% \mathrm{x}(\Delta$ prix $\mathrm{HT}) \mathrm{xI}_{\Delta \text { prix }}$

avec

$\mathrm{I}_{\Delta \text { prix }}=1 \mathrm{si} \mid \Delta$ prix $\mid>10 \%$

$I_{\Delta p r i x}=0$ sinon

Connaissant l'évolution du cours du brut, il est possible de simuler le prix toutes taxes comprises du carburant avec et sans TIPP flottante. Il en ressort d'abord que l'évolution du prix TTC des carburants est moins ample que celle du prix hors taxe, du fait que la TIPP porte sur les quantités (graphique 5).

Il apparaît clairement que si la TIPP flottante a eu pour effet de lisser quelque peu les prix, son effet sur les montants collectés est négligeable: les deux courbes de prix sans ce mécanisme (simulé) et avec (graphique 5) sont pratiquement identiques. C'est sans doute ce qui a conduit à retirer ce mécanisme depuis le 21 juillet 2002. 


\section{Graphique 3 Comparaison des prix avec instauration de la TIPP flottante}

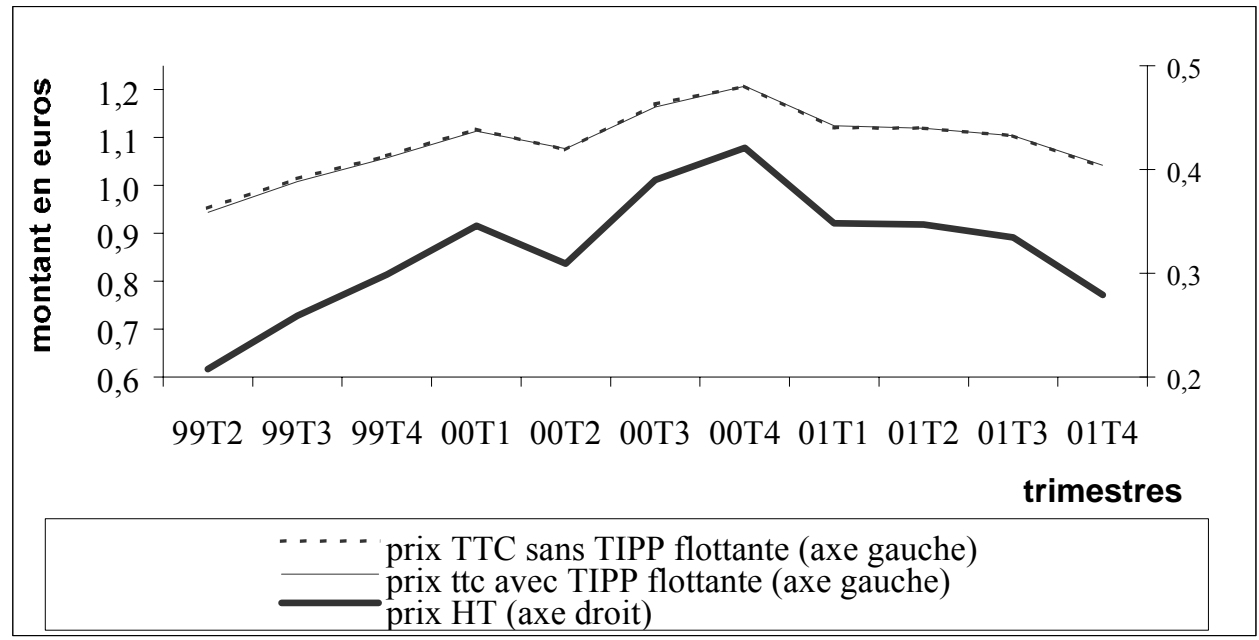

Source : Les Notes Bleues de Bercy; Projet de loi de finances pour 2001

\subsection{Les autres droits indirects}

\subsubsection{La taxe sur les conventions d'assurances}

Tout contrat d'assurance souscrit par une personne morale ou physique supporte, en substitution de la TVA, une taxe proportionnelle au montant hors taxe de la police d'assurance, appelée taxe sur les conventions d'assurances (art 991 du CGI):

$$
\text { prix toutes taxes comprises }=\left(1+t^{\prime}\right) \times \text { prix hors taxe }
$$

Le montant de $t$ ' varie en fonction du type d'assurance contractée tel que les assurances automobiles, incendie, dommages, etc..

\section{Tableau 6. Taux de taxation des différents contrats d'assurance}

\begin{tabular}{|l|r|}
\hline \multicolumn{1}{|c|}{ Type de contrat } & \multicolumn{2}{c|}{ Montant de la taxe } \\
\hline Assurance automobile & $18,00 \%$ \\
\hline Assurance incendie & $30 \%+3 €$ \\
\hline Assurance dommage- ouvrage & $21,5 \%+3 €$ \\
\hline Assurance maritime & $19 \%+3 €$ \\
\hline Assurance santé & $9 \%$ (jusqu'au $30 / 09 / 2002)$ \\
\hline Autres assurances & $9 \%+3 €$ \\
\hline
\end{tabular}

Source : www. ffsa. fr (Fédération Française des Sociétés d'assurance)

\subsubsection{La taxe sur les bijoux}

Les ventes de bijoux subissent en sus de la TVA depuis le $1^{\mathrm{er}}$ janvier 2000 une taxe de 4,5\% lorsque leur montant excède 3050 euros; dans le cas où ce montant serait compris entre 3050 
euros et 4600 euros, la base d'imposition est réduite d'un montant égal à la différence entre 4600 euros et ledit montant (article 150V bis du Code Général des Douanes).

\section{Graphique 4. Mécanisme d'imposition des bijoux}

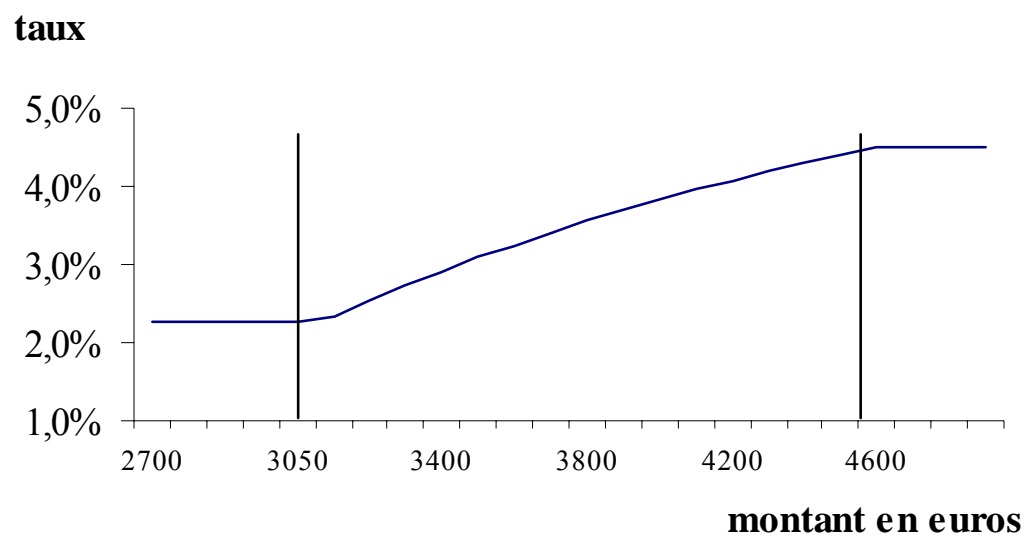

Source : www. douane.gouv.fr (MinéfiDgdi)

Pour un bijou d'une valeur de $3800 €$ hors taxe, la base d'imposition sera égale à $3000 €$.

$3800-(4600-3800)=3000$.

La taxation de ce bijou sera donc de $135 €:(3000 \times 0,045)=135$.

\subsubsection{La taxe sur les jeux de hasard}

Les jeux de hasard et d'argent constituent un phénomène majeur sur le plan sociologique, économique et budgétaire : $62 \%$ des Français jouent au moins une fois par an, dont 20\% régulièrement (Rapport du Sénat 223, 2001-2002).

Tableau 7. Taux de prélèvement de l'État sur les jeux de hasard

\begin{tabular}{|l|c|c|r|}
\hline \multicolumn{2}{|c|}{$\begin{array}{c}\text { Taux de prélèvement sur les mises, } \\
\text { en 2000 }\end{array}$} & $\begin{array}{c}\text { Montant des recettes } \\
\text { (en milliards d'euros) }\end{array}$ & \% des recettes \\
\hline Française Des Jeux & $26,0 \%$ & 1,8 & $46 \%$ \\
\hline Pari Mutuel Urbain & $15,7 \%$ & 1 & $26 \%$ \\
\hline Casinos & $53,0 \%$ & 1,1 & $\mathbf{2 8 \%}$ \\
\hline \multicolumn{2}{|c|}{ TOTAL } & & $\mathbf{3 , 9}$ \\
\hline
\end{tabular}

Source : Les jeux de hasard et d'argent en France : L'Etat croupier, le Parlement croupion ? Commission des finances, Les rapports du Sénat, $n^{\circ} 223$, 2001-2002 (p 192)

Les recettes perçues sur les jeux de hasard représentent 3,9 milliards d'euros en 2001 et les taux de prélèvements les plus importants (53\%) concernent les casinos. 


\section{La redistribution instaurée par la fiscalité indirecte sur les ménages}

L'étude de la redistribution s'appuie sur l'enquête Budget de Famille 2001, et notamment sur la nomenclature des dépenses, afin d'affecter à chaque ménage un montant de fiscalité indirecte selon sa consommation. Après avoir présenté les intérêts de cette source, cette partie s'attachera à mettre en relief l'impact différencié de la fiscalité indirecte au sein des ménages.

2.1 Les caractéristiques de l'enquête Budget De Famille 2001.

Les enquêtes «Budget de Famille » (BDF) décrivent avec détail l'ensemble des dépenses et des ressources des ménages. Ces dépenses sont ventilées dans une nomenclature d'environ 700 postes budgétaires. Toutes les dépenses sont couvertes, y compris celles qui ne relèvent pas de la consommation des biens et services au sens des Comptes Nationaux, notamment les impôts et taxes, ainsi que les consommations qui ne donnent pas lieu à dépense (autoconsommation alimentaire). L'édition 2001 de cette enquête a par ailleurs innové en collectant des informations sur les quantités, essentielles pour le calcul de certaines taxes à la consommation, ainsi que les types de magasins et les dates d'achat.

Outre les dépenses, l'étude des ressources est devenue un autre objectif de l'enquête, notamment depuis 1995. L'enquête Budget enregistre toutes les formes de ressources: revenus imposables ou non, prestations sociales reçues, sommes d'autres ménages, ressources exceptionnelles (loto, héritage, prime de licenciement,...).

Pour cette étude, les taux de TVA actuels et passés ont été introduits sur chaque poste élémentaire de la nomenclature de dépenses, afin de rendre possibles des analyses dans la dimension temporelle. La plupart des autres droits indirects sont intégrés, notamment ceux portant sur les carburants, les tabacs et les alcools.

Enfin, seules sont considérées les dépenses monétaires réalisées par les ménages, et non pas les dépenses de consommation au sens de la Comptabilité nationale. A ce titre, sont pris en compte les travaux d'entretien (comptabilisés en formation brute de capital fixe) soumis à TVA, mais pas l'autoconsommation alimentaire. 
2.2 La fiscalité indirecte présente un effet anti-redistributif marqué

La fiscalité indirecte représente un prélèvement élevé : En 2001, un ménage s'acquitte en moyenne d'un montant de $2640 €$ au titre de la fiscalité indirecte, soit 8\% du revenu disponible brut (cf. encadré 3). La TVA représente plus des deux tiers de cette somme. La TIPP arrive en seconde position, et représente près de $20 \%$ du total de la fiscalité indirecte. Les $2 / 3$ de la taxe sur les produits pétroliers sont prélevés sur l'essence, alors que le parc automobile est constitué pour moitié de véhicules diesel. Outre les carburants, l'automobile supporte aussi une taxe non négligeable sur les contrats d'assurance. Les autres taxes représentent environ $10 \%$ des recettes, la principale étant constituée des taxes sur les tabacs et alcools.

Tableau 8. La structure de la fiscalité indirecte sur les ménages en 2001

\begin{tabular}{|c|c|c|c|}
\hline & $\begin{array}{c}\text { Montant } \\
\text { moyen annuel } \\
(\text { en } €) \\
\end{array}$ & $\begin{array}{c}\text { Structure } \\
\text { (en\%) }\end{array}$ & $\begin{array}{l}\text { Taux d'effort } \\
\text { moyen (en \%) }\end{array}$ \\
\hline TVA dont & 1765 & 66,2 & 5,3 \\
\hline $\begin{array}{l}\text { TVA } 19,6 \% \\
\text { TVA } 5,5 \%\end{array}$ & $\begin{array}{c}1534 \\
231 \\
\end{array}$ & $\begin{array}{l}86,9 \\
13,1 \\
\end{array}$ & $\begin{array}{l}4,6 \\
0,7\end{array}$ \\
\hline TIPP dont & 515 & 19,5 & 1,6 \\
\hline $\begin{array}{l}\text { Essence } \\
\text { Diesel } \\
\end{array}$ & $\begin{array}{l}345 \\
168 \\
\end{array}$ & $\begin{array}{l}66,9 \\
32,7 \\
\end{array}$ & $\begin{array}{l}1,1 \\
0,4 \\
\end{array}$ \\
\hline Divers dont & 359,1 & 13,6 & 1,1 \\
\hline $\begin{array}{l}\text { Assurances auto } \\
\text { Alcools } \\
\text { Tabacs } \\
\text { Jeux de hasard } \\
\text { Autres (bijoux, assurances } \\
\text { hors automobile) }\end{array}$ & $\begin{array}{l}122 \\
36,7 \\
97,1 \\
16,6 \\
87,2\end{array}$ & $\begin{array}{c}33,8 \\
10,2 \\
27,0 \\
4,6 \\
24,3\end{array}$ & $\begin{array}{l}0,4 \\
0,1 \\
0,3 \\
0,1 \\
0,3\end{array}$ \\
\hline Total & $2639 €$ & 100 & 8,0 \\
\hline
\end{tabular}

Source : calculs sur l'enquête BDF 2001.

\section{Encadré 3. Définitions}

Le revenu disponible brut comprend les revenus d'activité, de remplacement, les prestations sociales et les revenus du patrimoine (y compris les loyers fictifs) avant tout prélèvement fiscal. 
Le revenu disponible net est égal au revenu disponible brut minoré de la CSG/CRDS, de la taxe d'habitation, de la taxe foncière, et de l'impôt sur le revenu des personnes physiques.

Le niveau de vie est égal au revenu disponible net par unité de consommation (échelle OCDE/INSEE).

Le niveau de vie est traditionnellement utilisé dans l'analyse des effets redistributifs comme une mesure du revenu corrigé de la structure démographique du ménage.

Le taux d'effort pour un prélèvement donné est égal au montant de ce prélèvement rapporté au revenu disponible brut.

L'utilisation du revenu disponible brut dans la mesure du taux d'effort résulte de la nécessité de tenir compte du poids relatif des différentes composantes de la fiscalité. Par ailleurs, comme la fiscalité indirecte porte sur les dépenses de consommation, il importe que les revenus de transferts perçus par les ménages soient pris en compte dans leurs ressources.

Le montant moyen de fiscalité indirecte acquitté par les ménages augmente naturellement avec le niveau de vie, mais dans une proportion moindre, compte tenu des structures de consommation. Il dépend aussi du montant des ressources, la propension à épargner étant croissante avec le revenu. En conséquence, le poids des taxes indirectes est 3,5 fois plus élevé dans le budget des ménages appartenant au premier décile de niveau de vie, comparativement à ceux des déciles supérieurs. Ces droits indirects représentent en moyenne près de $12 \% \mathrm{du}$ revenu disponible brut au sein des ménages les plus modestes, contre 3,3\% pour les plus aisés. Cet effet anti redistributif est compensé en grande partie par la fiscalité directe via la contribution sociale généralisée, et surtout par l'impôt sur le revenu (tableau 9).

Le poids de la fiscalité indirecte est accentué par un effet d'assiette : ces taxes touchent en effet tous les ménages, dès lors qu'ils consomment des biens et des services marchands. $A$ contrario, seule la moitié des ménages environ acquitte l'impôt sur le revenu, pour un montant moyen de $1900 €$ en 2001 calculé sur l'ensemble des ménages, qui atteint $2800 €$ par ménage imposé. L'impôt sur le revenu représente ainsi un taux d'effort moyen de 4,6\% par ménage, soit près de deux fois inférieur à celui de la fiscalité indirecte. 
Tableau 9: Comparaison des taux d'effort entre fiscalité directe et indirecte

\begin{tabular}{|l|c|c|c|c|c|c|}
\hline \multicolumn{2}{|c|}{} & \multicolumn{5}{|c|}{ Taux d'effort (en \% du revenu disponible brut) } \\
\hline $\begin{array}{c}\text { Decile de } \\
\text { niveau de } \\
\text { vie }\end{array}$ & $\begin{array}{c}\text { Montant } \\
\text { moyen de } \\
\text { fiscalité } \\
\text { indirecte en } €\end{array}$ & $\begin{array}{c}\text { Fiscalité } \\
\text { indirecte }\end{array}$ & CSG-CRDS & $\begin{array}{c}\text { Taxes } \\
\text { d'habitation } \\
\text { et foncière }\end{array}$ & $\begin{array}{c}\text { Impôt sur le } \\
\text { revenu }\end{array}$ & Total \\
\hline 01 & 1841 & 11,7 & 3,8 & 1,7 & 0,8 & 18,0 \\
02 & 2298 & 9,8 & 5,0 & 2,0 & 1,7 & 18,5 \\
03 & 2687 & 9,0 & 5,8 & 2,2 & 2,6 & 19,6 \\
04 & 2935 & 8,1 & 6,1 & 2,3 & 3,8 & 20,3 \\
05 & 3292 & 7,5 & 6,4 & 2,2 & 5,0 & 21,1 \\
06 & 3343 & 6,6 & 6,5 & 2,4 & 6,5 & 22,0 \\
07 & 3496 & 6,2 & 6,6 & 2,3 & 7,1 & 22,2 \\
08 & 3830 & 5,7 & 6,8 & 2,0 & 8,0 & 22,5 \\
09 & 3927 & 4,8 & 6,8 & 2,2 & 9,6 & 23,4 \\
10 & 4235 & 3,3 & 7,0 & 1,5 & 12,0 & 23,8 \\
\hline Ensemble & $\mathbf{2 6 3 9}$ & $\mathbf{8 , 0}$ & $\mathbf{5 , 9}$ & $\mathbf{2 , 1}$ & $\mathbf{4 , 6}$ & $\mathbf{2 0 , 6}$ \\
\hline
\end{tabular}

Source : calculs à partir de l'enquête Budget 2001

2.3 Le poids de la fiscalité indirecte selon le type de ménage

En structure, la part de la fiscalité indirecte varie sensiblement selon la composition du ménage. Les montants dépensés sont de plus en plus élevés à mesure que la taille de la famille s'accroît. Les femmes seules se distinguent par un poids de la TVA nettement plus élevé : elles consomment relativement plus de produits frais que les autres ménages et ont davantage recours à des services. Les femmes seules, généralement de plus de 50 ans, dépensent moins en carburant du fait qu'elles ne possèdent pas nécessairement de véhicule automobile. A l'inverse, pour les hommes seuls, le poste transports se détache nettement, au travers de la consommation de carburant et des taxes liées aux assurances automobiles. Le poids de la TIPP tend à décroître légèrement avec le nombre d'enfants. Pour les familles monoparentales comme pour les hommes seuls, une part plus importante de leur budget est consacrée à la consommation de tabac.

Considéré en fonction du niveau de vie, les poids respectifs des composantes de la fiscalité indirecte présentent de très fortes disparités (tableau 10b). Les ménages à faible niveau de vie contribuent relativement plus au titre des dépenses sur les produits pétroliers, les alcools, et le tabac et relativement moins au titre de la seule TVA que les ménages à niveau de vie élevé (qu'il s'agisse de la TVA à taux réduit ou normal). 
Tableau 10a Structure de la fiscalité indirecte par type de ménage (en \%)

\begin{tabular}{|c|c|c|c|c|c|c|c|c|}
\hline & $\begin{array}{c}\text { Total } \\
\text { ménage }\end{array}$ & $\begin{array}{c}\text { homme } \\
\text { seul }\end{array}$ & $\begin{array}{c}\text { femme } \\
\text { seule }\end{array}$ & $\begin{array}{c}\text { famille } \\
\text { mono } \\
\text { parentale }\end{array}$ & $\begin{array}{c}\text { couple } 0 \\
\text { enfant }\end{array}$ & $\begin{array}{l}\text { couple } 1 \\
\text { enfant }\end{array}$ & $\begin{array}{l}\text { couple } 2 \\
\text { enfants }\end{array}$ & $\begin{array}{l}\text { couple } 3 \\
\text { enfants }\end{array}$ \\
\hline TVA dont & 66,9 & 63,4 & 73,2 & 67,5 & 66,5 & 65,6 & 66,8 & 67,3 \\
\hline TVA $19,6 \%$ & 58,1 & 55,9 & 63,1 & 58,4 & 57,2 & 57,6 & 58,4 & 58,5 \\
\hline TVA 5,5\% & 8,8 & 7,5 & 10,2 & 9,1 & 9,3 & 8,1 & 8,4 & 8,9 \\
\hline TIPP dont & 19,5 & 21,1 & 13,8 & 17,9 & 19,5 & 20,9 & 20,7 & 19,5 \\
\hline Essence & 13,1 & 13,4 & 10,9 & 13,9 & 13,6 & 13,1 & 13,7 & 11,4 \\
\hline Divers dont & 13,6 & 15,6 & 12,9 & 14,6 & 14,0 & 13,4 & 12,5 & 13,1 \\
\hline Assurance auto & 4,6 & 4,7 & 3,7 & 4,1 & 5,0 & 4,8 & 4,5 & 4,3 \\
\hline Tabacs & 3,7 & 5,0 & 2,9 & 5,6 & 2,6 & 4,2 & 3,7 & 4,0 \\
\hline Alcools & 1,4 & 1,6 & 1,2 & 1,4 & 1,8 & 1,1 & 1,2 & 1,3 \\
\hline Autres & 3,9 & 4,3 & 5,2 & 3,5 & 4,6 & 3,3 & 3,2 & 3,5 \\
\hline Total & 100 & 100 & 100 & 100 & 100 & 100 & 100 & 100 \\
\hline $\begin{array}{c}\text { Montant moyen } \\
\text { en euros }\end{array}$ & 2639 & 1951 & 1362 & 2246 & 2635 & 3591 & 3768 & 3904 \\
\hline
\end{tabular}

Source : calculs à partir de l'enquête BDF 2001

Tableau 10b Structure de la fiscalité indirecte par décile de niveau de vie (en \%)

\begin{tabular}{|c|c|c|c|c|c|c|c|c|c|c|c|}
\hline $\begin{array}{r}\text { Décile de niveau } \\
\text { de vie }\end{array}$ & total & 01 & 02 & 03 & 04 & 05 & 06 & 07 & 08 & 09 & 10 \\
\hline TVA dont & 66,9 & 65,8 & 65,5 & 65,4 & 65,3 & 65,5 & 65,1 & 67,0 & 67,5 & 69,7 & 71,7 \\
\hline TVA 19,6\% & 58,1 & 56,8 & 56,1 & 56,2 & 56,3 & 56,4 & 56,5 & 58,4 & 58,9 & 61,4 & 63,8 \\
\hline TVA 5,5\% & 8,8 & 9,1 & 9,4 & 9,2 & 9,0 & 9,1 & 8,6 & 8,6 & 8,6 & 8,3 & 7,9 \\
\hline TIPP dont & 19,5 & 18,7 & 19,1 & 19,3 & 20,0 & 20,6 & 21,1 & 20,2 & 20,0 & 18,4 & 16,8 \\
\hline Essence & 13,1 & 12,2 & 12,9 & 13,0 & 13,8 & 13,5 & 13,9 & 12,9 & 13,1 & 12,6 & 12,2 \\
\hline Divers Dont & 13,6 & 15,5 & 15,4 & 15,2 & 14,7 & 13,9 & 13,8 & 12,8 & 12,5 & 11,9 & 11,4 \\
\hline Alcools & 1,4 & 1,4 & 1,3 & 1,4 & 1,3 & 1,6 & 1,5 & 1,3 & 1,3 & 1,3 & 1,5 \\
\hline Tabacs & 3,7 & 6,1 & 5,2 & 4,7 & 4,3 & 3,5 & 3,9 & 3,0 & 2,7 & 2,5 & 1,9 \\
\hline Autres & 8,5 & 8,1 & 8,8 & 9,1 & 9,0 & 8,8 & 8,4 & 8,5 & 8,6 & 8,2 & 8,0 \\
\hline Total & 100 & 100 & 100 & 100 & 100 & 100 & 100 & 100 & 100 & 100 & 100 \\
\hline
\end{tabular}

Source : calculs à partir de l'enquête BDF 2001

Les analyses sur les structures sont confortées par celles des taux d'effort. Ceux-ci sont d'autant plus élevés que la taille de la famille augmente. Ils sont en revanche inférieurs à la moyenne pour les personnes seules, les familles monoparentales et les couples sans enfant. La présence d'enfants augmente donc sensiblement la contribution moyenne au titre de la fiscalité indirecte. 
Le taux d'effort induit par la fiscalité indirecte diminue fortement avec l'augmentation du niveau de vie (tableau 11b) : il varie de 1 à 3 entre les ménages les plus aisés et les plus modestes. Cette disparité est particulièrement accentuée pour les taxes diverses (tabacs, alcools), ainsi que pour les droits sur le produits pétroliers (TIPP). Les disparités sont également plus fortes sur la TVA à taux réduit en regard de celle à taux normal.

Tableau 11a : Taux d'effort des impôts indirects par type de ménage (en \%)

\begin{tabular}{|c|c|c|c|c|c|c|c|c|}
\hline & $\begin{array}{c}\text { Total } \\
\text { ménage }\end{array}$ & $\begin{array}{c}\text { homme } \\
\text { seul }\end{array}$ & $\begin{array}{c}\text { femme } \\
\text { seule }\end{array}$ & $\begin{array}{c}\text { famille } \\
\text { mono } \\
\text { parentale }\end{array}$ & $\begin{array}{c}\text { couple } 0 \\
\text { enfant }\end{array}$ & $\begin{array}{c}\text { couple } 1 \\
\text { enfant }\end{array}$ & $\begin{array}{c}\text { couple } 2 \\
\text { enfants }\end{array}$ & $\begin{array}{c}\text { couple } 3 \\
\text { enfants }\end{array}$ \\
\hline TVA dont & 5,3 & 5,1 & 5,1 & 5,8 & 5,0 & 5,5 & 5,7 & 5,8 \\
\hline TVA 19,6\% & 4,6 & 4,5 & 4,4 & 5,0 & 4,3 & 4,8 & 4,9 & 5,0 \\
\hline TVA 5,5\% & 0,7 & 0,6 & 0,7 & 0,8 & 0,7 & 0,7 & 0,7 & 0,8 \\
\hline TIPP dont & 1,6 & 1,7 & 1,0 & 1,5 & 1,5 & 1,8 & 1,8 & 1,7 \\
\hline Essence & 1,0 & 1,1 & 0,8 & 1,2 & 1,0 & 1,1 & 1,2 & 1,0 \\
\hline Divers dont & 1,1 & 1,3 & 0,9 & 1,2 & 1,1 & 1,1 & 1,1 & 1,1 \\
\hline Assurance auto & 0,4 & 0,4 & 0,3 & 0,4 & 0,4 & 0,4 & 0,4 & 0,4 \\
\hline Tabacs & 0,3 & 0,4 & 0,2 & 0,5 & 0,2 & 0,4 & 0,3 & 0,3 \\
\hline Alcools & 0,1 & 0,1 & 0,1 & 0,1 & 0,1 & 0,1 & 0,1 & 0,1 \\
\hline Autres & 0,3 & 0,3 & 0,4 & 0,3 & 0,3 & 0,3 & 0,3 & 0,3 \\
\hline Total & 8,0 & 8,1 & 7,0 & 8,5 & 7,5 & 8,4 & 8,5 & 8,6 \\
\hline RDB & 33020 & 24175 & 19549 & 26302 & 35087 & 42781 & 44455 & 45210 \\
\hline
\end{tabular}

Source : calcul à partir de l'enquête BDF 2001

Tableau 11b : Taux d'effort des impôts indirects par décile de niveau de vie (en \%)

\begin{tabular}{|l|c|c|c|c|c|c|c|c|c|c|c|}
\hline $\begin{array}{l}\text { Déciles de niveau } \\
\text { de vie }\end{array}$ & total & 01 & 02 & 03 & 04 & 05 & 06 & 07 & 08 & 09 & 10 \\
\hline & & & & & & & & & & & \\
Tva dont & $\mathbf{5 , 3}$ & $\mathbf{8 , 1}$ & $\mathbf{7 , 1}$ & $\mathbf{6 , 7}$ & $\mathbf{6 , 2}$ & $\mathbf{5 , 9}$ & $\mathbf{5 , 6}$ & $\mathbf{5 , 3}$ & $\mathbf{5 , 0}$ & $\mathbf{4 , 4}$ & $\mathbf{3 , 4}$ \\
TVA 19,6\% & 4,6 & 7,0 & 6,1 & 5,7 & 5,3 & 5,0 & 4,9 & 4,6 & 4,3 & 3,9 & 3,0 \\
TVA 5,5\% & 0,7 & 1,1 & 1,0 & 0,9 & 0,9 & 0,8 & 0,7 & 0,7 & 0,6 & 0,5 & 0,4 \\
\hline & & & & & & & & & & & \\
TIPP dont & $\mathbf{1 , 6}$ & $\mathbf{2 , 3}$ & $\mathbf{2 , 1}$ & $\mathbf{2 , 0}$ & $\mathbf{1 , 9}$ & $\mathbf{1 , 8}$ & $\mathbf{1 , 8}$ & $\mathbf{1 , 6}$ & $\mathbf{1 , 5}$ & $\mathbf{1 , 2}$ & $\mathbf{0 , 8}$ \\
Essence & 1,0 & 1,5 & 1,4 & 1,3 & 1,3 & 1,2 & 1,2 & 1,0 & 1,0 & 0,8 & 0,6 \\
\hline & & & & & & & & & & & \\
Divers dont & $\mathbf{1 , 1}$ & $\mathbf{1 , 9}$ & $\mathbf{1 , 7}$ & $\mathbf{1 , 6}$ & $\mathbf{1 , 4}$ & $\mathbf{1 , 2}$ & $\mathbf{1 , 2}$ & $\mathbf{1 , 0}$ & $\mathbf{0 , 9}$ & $\mathbf{0 , 8}$ & $\mathbf{0 , 5}$ \\
Alcools & 0,3 & 0,2 & 0,1 & 0,1 & 0,1 & 0,1 & 0,1 & 0,1 & 0,1 & 0,1 & 0,1 \\
Tabacs & 0,1 & 0,7 & 0,6 & 0,5 & 0,4 & 0,3 & 0,3 & 0,2 & 0,2 & 0,2 & 0,1 \\
Autres & 0,7 & 1,0 & 0,9 & 0,9 & 0,8 & 0,8 & 0,7 & 0,7 & 0,6 & 0,5 & 0,4 \\
\hline TOTAL Fisc Ind. & $\mathbf{8 , 0}$ & $\mathbf{1 2 , 4}$ & $\mathbf{1 0 , 8}$ & $\mathbf{1 0 , 2}$ & $\mathbf{9 , 4}$ & $\mathbf{8 , 9}$ & $\mathbf{8 , 6}$ & $\mathbf{7 , 9}$ & $\mathbf{7 , 3}$ & $\mathbf{6 , 3}$ & $\mathbf{4 , 7}$ \\
\hline
\end{tabular}




\subsection{Un effet anti-redistributif relativement élevé}

L'effet de la fiscalité indirecte sur les niveaux de vie peut être visualisé à travers une courbe de Lorenz tracée avant et après la prise en compte des prélèvements indirects. Avant toute taxe, le $1^{\text {er }}$ décile de niveau de vie cumule $3,9 \%$ du revenu net total, tandis que le dernier décile compte pour $24,1 \%$ de ce revenu. Après fiscalité indirecte, ces parts s'élèvent respectivement à $3,6 \%$ et $24,8 \%$.

\section{Graphique 5 : Perte de revenu disponible net liée à la fiscalité indirecte (en \%)}

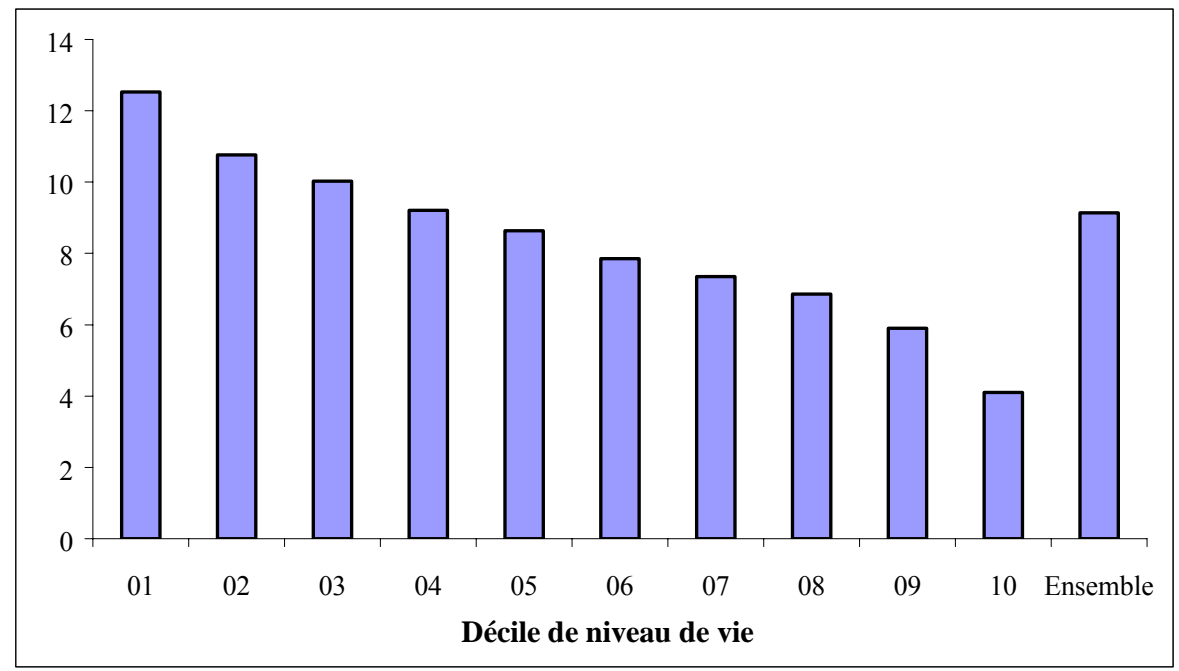

Source : calculs à partir de l'enquête BDF 2001

La fiscalité indirecte conduit ainsi à une distribution des niveaux de vie plus inégalitaire. Le coefficient de Gini s'accroît de 1,3 point, de 0,274 avant toute taxe, à 0,287 après. Le $1^{\text {er }}$ décile de niveau de vie subit une perte de $12,5 \%$ de son revenu disponible net alors que le dernier décile n'est touché qu'à hauteur de 4,1\%. Les 3 premiers déciles supportent le plus les taxes sur la consommation, avec plus de $10 \%$ de perte de revenu disponible net. Pour les 3 derniers déciles en revanche, le taux de prélèvement est inférieur à 7 points.

Cet effet anti-redistributif de la fiscalité indirecte peut être mis en perspective avec la totalité de la redistribution entre le revenu initial et le revenu disponible net des ménages. Cette redistribution opérée par la fiscalité directe, les prestations familiales, les aides à la scolarité, l'allocation logement, et les minima sociaux conduit à une diminution du coefficient de Gini d'environ 8 points (France Portrait social, édition 2002-2003). 


\section{Graphique 6. Distribution du revenu net avant et après les prélèvements indirects}

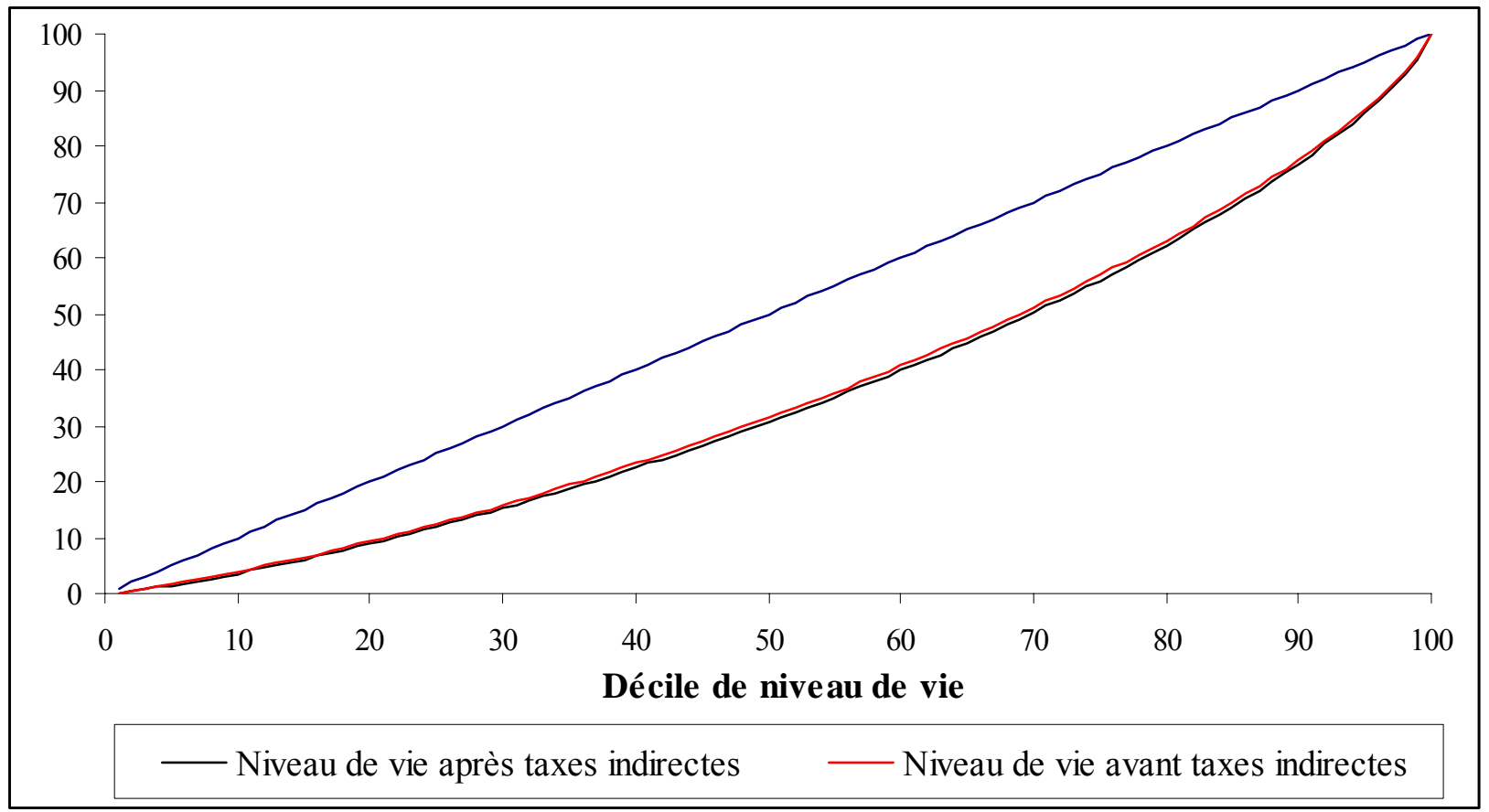

Source : calculs à partir de l'enquête BDF 2001

\section{L'impact de l'évolution des taux de TVA entre 1976 et 2001 sur la} répartition de revenu : une analyse à structure constante

Il est possible, avec la base de données constituée, d'analyser les effets des modifications de la législation de la TVA intervenues au cours des dernières décennies. Les changements successifs des taux de la TVA depuis 1976 ont été appliqués à la structure de la consommation donnée par l'enquête Budget 2001 (tableau 19). Cette démarche permet d'isoler l'effet des seuls changements de taux parmi tous les autres facteurs conduisant à des variations de recettes de TVA (évolution des revenus, des prix, des structures de consommation, etc.).

En application de la législation de 1976, les ménages appartenant au $1^{\text {er }}$ décile de niveau de vie acquittent un montant de TVA de $1422 €$, ce qui représente un taux moyen d'imposition de 9,1\% (6,8\% pour l'ensemble des ménages). En 1982, l'instauration d'un taux réduit sur les produits de l'alimentation et surtout la fusion des taux normal et intermédiaire à $18,6 \%$ bénéficie plutôt aux déciles de niveau de vie intermédiaires, les taux de prélèvement ne diminuant que légèrement pour les autres. 
Tableau 12 : Évolution du taux d'effort induit par la TVA selon le décile de niveau de vie en \%

\begin{tabular}{|c|c|c|c|c|c|c|c|}
\hline $\begin{array}{c}\text { Décile de } \\
\text { niveau de } \\
\text { vie }\end{array}$ & $\mathbf{1 9 7 6}$ & $\mathbf{1 9 8 2}$ & $\mathbf{1 9 8 9}$ & $\mathbf{1 9 9 2}$ & $\mathbf{1 9 9 3}$ & $\mathbf{1 9 9 5}$ & $\mathbf{2 0 0 1}$ \\
\hline 01 & 9,1 & 8,9 & $\mathbf{8 , 5}$ & 8,2 & 7,7 & 8,3 & 7,7 \\
02 & 8,0 & 7,8 & 7,4 & 7,1 & 6,4 & 6,9 & 6,4 \\
03 & 7,5 & 7,0 & 6,6 & 6,4 & 5,9 & 6,3 & 5,8 \\
04 & 7,1 & 6,7 & 6,3 & 6,1 & 5,4 & 5,9 & 5,4 \\
05 & 6,7 & 6,2 & 5,8 & 5,6 & 5,1 & 5,5 & 5,0 \\
06 & 5,9 & 5,5 & 5,1 & 4,9 & 4,7 & 5,1 & 4,6 \\
07 & 5,5 & 5,1 & 4,9 & 4,7 & 4,3 & 4,7 & 4,3 \\
08 & 5,0 & 4,8 & 4,5 & 4,4 & 4,1 & 4,4 & 4,1 \\
09 & 4,2 & 3,9 & 3,7 & 3,6 & 3,5 & 3,8 & 3,5 \\
10 & 3,0 & 2,8 & 2,7 & 2,5 & 2,4 & 2,6 & 2,4 \\
\hline Ensemble & $\mathbf{6 , 8}$ & $\mathbf{6 , 4}$ & $\mathbf{6 , 1}$ & $\mathbf{5 , 8}$ & $\mathbf{5 , 4}$ & $\mathbf{5 , 8}$ & $\mathbf{5 , 3}$ \\
\hline Ratio & 3,03 & 3,18 & 3,15 & 3,28 & 3,21 & 3,19 & 3,2 \\
D1/D10 & & &
\end{tabular}

Source : calculs à partir de l'enquête BDF 2001

En 1989, les boissons non alcoolisées, auparavant taxées au taux intermédiaire, sont ramenées au taux super réduit de 5,5\%. Cette modification a le plus bénéficié aux premiers déciles qui, de part leur structure de consommation, consacrent une grande part de leur budget à l'alimentation.

En 1992, le taux majoré a été abaissé de 28 à 22\%. Cette mesure a bénéficié en moyenne aux premiers déciles mais leur taux d'effort reste toujours supérieur à la moyenne. La suppression du taux majoré au $1^{\text {er }}$ janvier 1993 conduit également à une diminution des taux d'effort plus favorable aux premiers déciles. En 1995, le taux normal passe de 18,6 à 20,6\%. Tous les ménages sont affectés par cette hausse mais pas de la même manière : le taux d'effort des ménages des deux premiers déciles s'accroît de plus de 0,5 point, contre moins de 0,3 pour les deux derniers.

Au total, sur la période 1976-2000, les baisses de TVA ont globalement conduit à une baisse du taux d'effort pour l'ensemble des ménages, de 1,5 point environ. Toutefois, ce taux demeure 3 fois supérieur pour le premier décile relativement au dernier. Ainsi, la baisse générale des taux de TVA au cours des dernières décennies n'a pas conduit à en répartir différemment la charge selon les niveaux de vie. 


\section{Détermination du montant d'impôt indirect en fonction des caractéristiques du ménage.}

$\mathrm{Au}$ vu des premiers tableaux statistiques, le montant acquitté au titre de la fiscalité indirecte semble être pour partie lié aux différentes caractéristiques des ménages, outre l'effet revenu. Afin d'isoler chacun de ces effets, une équation de régression a été construite pour la fiscalité indirecte dans son ensemble, puis pour certaines de ses composantes.

\subsection{Une prédominance de l'effet revenu pour la fiscalité indirecte}

L'effet revenu pèse fortement sur la charge de la fiscalité indirecte : le volume et la structure de la consommation, et par voie de conséquence les taxes y afférant, augmente bien évidemment avec le revenu. Cependant, l'élasticité du montant total de fiscalité indirecte au revenu est inférieure à l'unité. Une augmentation du revenu de 10\% entraînerait ainsi une augmentation de $4 \%$ des recettes de fiscalité indirecte. La charge de la fiscalité indirecte est donc, toutes choses égales par ailleurs, moins que proportionnelle au revenu.

Les montants d'impôts indirects acquittés augmentent avec le nombre d'enfants: un célibataire dépense moins qu'un couple avec enfant. Cet effet s'observe notamment pour le $1^{\text {er }}$ et le $3^{\mathrm{e}}$ enfant, il est atténué pour le second enfant.

L'effet diplôme n'est pas très significatif : certes, les sans diplôme acquittent moins de taxes que les diplômés, mais cet écart semble être essentiellement lié aux différences de revenus.

Les jeunes ménages se distinguent nettement du reste de la population. Les plus jeunes optent plus que leurs aînés pour la consommation de services, et pour des biens a priori davantage taxés. Ils supportent de ce fait un poids plus élevé de fiscalité indirecte.

Les agriculteurs, artisans et personnes sans profession dépensent moins comparativement aux ouvriers et employés. En revanche, les professions intermédiaires et les cadres paient relativement plus de taxes indirectes. Enfin, des différences régionales apparaissent. Les Parisiens, les habitants du bassin parisien, de l'Ouest et du pourtour méditerranéen supportent moins de fiscalité indirecte que ceux du Nord, du Sud-ouest et du Centre-est.

Ainsi pratiquement toutes les caractéristiques considérées ont un effet significatif, mais c'est surtout l'effet revenu qui explique les montants acquittés de fiscalité indirecte, avec une élasticité positive et inférieure à l'unité. 
4.2 Cet effet revenu varie sensiblement selon la taxe considérée

Ce modèle global de fiscalité indirecte peut masquer des différences importantes selon la nature de la taxe. Il importe donc de mener une analyse similaire pour chaque taxe. Mais dans ce cas, la procédure d'estimation doit être modifiée pour pallier d'éventuels biais de sélection induits par la non dépense. Celle-ci peut être liée à une non consommation effective du ménage, ou une absence d'achat durant l'enquête. Par exemple, pour un ménage, ne pas avoir de dépense en boissons alcoolisées peut correspondre à une non consommation de produits de cette nature, ou à la consommation du stock constitué avant les deux semaines d'enquête. Or, le fait de consommer ou de ne pas consommer de boissons alcoolisées est en partie lié aux caractéristiques sociodémographiques du ménage.

La procédure initiée par Heckman (Heckman, 1980, Deaton 1984)) en deux étapes a été utilisée pour prendre en compte le biais de sélection éventuel : Dans une première étape est estimée la probabilité de ne pas avoir consommé lors des 2 semaines de l'enquête, qu'il s'agisse d'une non consommation réelle ou liée au mode de collecte. Les variables de sélection retenues sont notamment la taille de la commune d'habitation, et le niveau de diplôme du chef de ménage.

Dans une seconde étape, le ratio de Mills est calculé à partir de la probabilité estimée et son inverse (appelé lambda) est introduit comme variable explicative de l'équation estimée. Sa significativité révèle la présence d'un biais de sélection. De fait, l'inverse du ratio de Mills (et donc l'effet de sélection), s'est avéré non significatif pour le tabac et les jeux de hasard, mais il l'est pour la TIPP et les dépenses d'alcool.

L'effet revenu est légèrement plus fort sur la TVA que pour l'ensemble de la fiscalité indirecte. L'élasticité revenu des montants de la TVA à taux réduit est moins élevée $(0,39)$ que celle de la TVA à taux normal $(0,46)$ du fait que le taux réduit est appliqué en général aux produits de première nécessité. Les élasticités revenu des taxes sur l'alcool et les jeux sont relativement faibles, respectivement égales à 0,16 et 0,13 . Une augmentation de $10 \%$ du revenu conduirait ainsi à un accroissement de 1,6\% des taxes collectées sur les alcools, et de 1,3\% pour celles portant sur les jeux. L'élasticité revenu des taxes sur les produits pétroliers est plus forte $(0,31)$. A l'inverse, les taxes payées sur le tabac sont pratiquement insensibles au revenu. L'élasticité estimée $(0,015)$ est proche de zéro et non significative. Une augmentation (baisse) du revenu n'entraînerait donc aucun changement dans la consommation de tabac. 
Tableau 13 : Mesure de l'élasticité du revenu selon le poste de fiscalité indirecte

\begin{tabular}{|l|c|}
\hline & élasticité revenu \\
\hline TVA totale & 0.44 \\
TVA à taux normal $(19,6 \%)$ & 0.46 \\
TVA à taux réduit $(5,5 \%)$ & 0.39 \\
TIPP & 0,31 \\
Alcool & 0.16 \\
Tabac & 0.01 \\
Jeux & 0,12 \\
\hline Ensemble Fiscalité indirecte & $\mathbf{0 . 4 3}$ \\
\hline
\end{tabular}

Source : calculs à partir l'enquête BDF 2001

Les caractéristiques sociodémographiques des ménages peuvent modifier légèrement l'impact de l'effet revenu. Les montants collectés de TVA varient très peu en fonction des caractéristiques de ménage. En revanche, les montants de taxes collectées sur les alcools sont plus élevés au sein des familles monoparentales et varient considérablement selon la région (opposition Nord Sud ) du fait des modes de production et des habitudes de consommation. Les taxes sur les carburants (TIPP) sont davantage acquittées par les jeunes de moins de 30 ans. Elles sont aussi plus importantes chez les hommes célibataires et beaucoup moins élevées chez les familles monoparentales. Par rapport aux personnes inactives, les montants de TIPP diminuent en fonction de la catégorie socioprofessionnelle, surtout pour les cadres. Les habitants de l'agglomération parisienne, toutes choses égales par ailleurs, roulent moins que les habitants des communes rurales.

Les dépenses consacrées au tabac et les taxes qui y sont associées diminuent à mesure que le diplôme est élevé : les montants acquittés par les femmes seules et les familles monoparentales sont inférieurs à ceux des hommes seuls. Par rapport aux ouvriers, les agriculteurs paient moins de taxes sur le tabac. Les habitants des grandes agglomérations dépensent plus en achat de cigarettes, par opposition aux habitants des communes rurales. Enfin, les montants des taxes associées aux jeux de hasard pèsent davantage sur les célibataires que sur les couples avec au moins un enfant. 


\section{CONCLUSION}

La constitution d'une base de donnés issue de l'Enquête Budget de Famille 2001 a permis d'introduire des taux de fiscalité indirecte à un niveau fin des dépenses pour chaque ménage. Il est alors possible de calculer des taux moyens d'imposition au titre des différentes taxes sur la consommation (TVA, TIPP, accises...), et d'étudier comment les montants se répartissent au sein des ménages. Le poids et la répartition des différentes taxes indirectes entre les ménages dépendent essentiellement des différences entre les structures de consommation, elles-mêmes liées au revenu disponible, et à la taille de la famille.

En conséquence, les effets redistributifs de ces taxes traitées globalement et séparément varient sensiblement selon les caractéristiques des ménages. Si en moyenne la part du revenu disponible brut consacrée à la fiscalité indirecte est de $8 \%$, elle varie de $11,7 \%$ pour le premier décile de niveau de vie à 3,3\% pour le dernier. Par son effet anti-redistributif la fiscalité indirecte contribue à augmenter les inégalités (mesurées par le coefficient de Gini) de plus d'un point.

Une analyse économétrique des montants payés en fonction des caractéristiques sociodémographiques du ménage a montré sans surprise un effet revenu prépondérant, avec une élasticité positive, mais inférieure à l'unité $(0,4)$, pour l'ensemble de la fiscalité indirecte. Toutefois, cet effet revenu varie sensiblement selon la nature de la taxation. Pour la TIPP, l'élasticité est proche de 0,3 . Pour les tabacs, l'élasticité des recettes au revenu est quasiment nulle. Enfin, pour les jeux et l'alcool, les élasticités au revenu sont relativement faibles, comprises entre 0,12 et 0,16 . 


\section{BIBLIOGRAPHIE}

Accardo J. 1997 « Les prélèvements socio-fiscaux en 1994 », Synthèses n¹1, pp. 15-25.

Amiel, A. 2003 «Les effets redistributifs de la fiscalité indirecte en France : une analyse à partir de l'enquête Budget de famille 2001 » Mémoire de DESS Méthodes quantitatives et modélisation pour l'entreprise de l'Université de la Réunion effectué à la Direction générale de l'INSEE à Paris.

Baccouche, R. et Laisney, F. 1986 « Analyse microéconomique de la réforme de la TVA de juillet 1982 en France », Annales d'économie et de statistiques, n², avril/juin 1986, pp. 37-74.

Baccouche, R. et Laisney, F.1990 «Simulation of value-added tax reforms for France using crosssection data » dans ,Microeconometrics : surveys applications, Basil Blackwell 1990, pp.265-301.

Bazy-Malaurie C., Coutière A., et Roux B. 1982 "La TVA dans la consommation des ménages », Economie et statistique, ${ }^{\circ} 150$, décembre 1982, pp. 17-27.

Bourguignon F. Fiscalité et redistribution 1998, Conseil d'Analyse Economique (CAE), n 11

Centre de documentation et d'information de l'assurance « Taxes et contributions sur les cotisations d'assurance » http://www.ffsa.fr

Commission des finances «Les jeux de hasard et d'argent en France : L'Etat croupier, le Parlement croupion? » Les rapports du Sénat, $n^{\circ} 223,2001-2002$ (p 192)

Conseil des impôts 1993 « La taxe sur la valeur ajoutée ».

David M-G., Lhommeau B., Starzec C. 1999 «Le modèle de Microsimulation INES: description et exemple d'utilisation », INSEE, Document de travail N F9902.

Deaton, A.S. and M. Irish (1984),"A Statistical Model for Zero Expenditures in Household Budgets" Journal of Public Economics, 23: 59-80.

« Du revenu initial au revenu disponible : le point sur le système socio-fiscal en 2001 » France Portrait social, édition 2002-2003, pp79-97.

Gardes F., Lhommeau B., Starzec C. 1998, «Introducing Behavioural Response into Microsimulation Model: Simulation of VAT Modifications on Consumers' Behaviour » communication au colloque Microsimulation in the new millenium-chalenges and innovations. Cambridge, Grande Bretagne, 
Heckman, J. 1990, "Varieties of Selection Bias (in Selectivity Bias: New Developments)", The American Economic Review, Vol. 80, No. 2, Papers and Proceedings of the Hundred and Second Annual Meeting of the American Economic Association, May, pp. 313-318.

INSEE, Comptes Nationaux 2000 « Rapport sur les comptes de la Nation».

Lefèbvre fiscal 1976 « Section VII. Taux de la TVA » Memento fiscal, pp 536-571

Lefèbvre fiscal 1984. «Titre 4. Les taux de la TVA » Memento fiscal, pp 81-105

Lefèbvre fiscal 1989.« Section 3. Taux de la TVA » Memento fiscal, pp 612-631

Lefèbvre fiscal 1993.« Section 3. Taux de la TVA » Memento fiscal, pp 661-671

Lefèbvre fiscal 1993.« Section 3. Taux de la TVA » Memento fiscal, pp 713-730

Ministère de l'Economie, des Finances et de l'Industrie- 09/2002 «Le Budget de l'État » http://www.minefi.gouv.fr/minefi/publique/budget_etat/index.htm

Ministère de l'Economie, des Finances et de l'Industrie «Régime fiscal des alcools et boissons alcooliques »

http://www.douane.gouv.fr/finc.asp?page=entreprise/d1210.htm\&cusnum=496

Ministère de l'Economie, des Finances et de l'Industrie " Régime fiscal et économique des tabacs » http://www.douane.gouv.fr/finc.asp?page=entreprise/d1202.htm\&cusnum=732

Murat F., Roth N., Starzec C 2002 «L'évolution de la redistribution du système socio- fiscal entre 1990 et 1998 : une analyse à structure constante» France, portrait social, 2000-2001

NICHELE V., ROBIN JM. 1993, «Evaluation des effets budgétaires et redistributifs de réformes de la fiscalité indirecte française » Economie et Prévision, $n^{\circ}$ 110-111, pp. 105- 128

PICARD N., RUIZ N., TRANNOY A. 2003 « Un modèle de microsimulation des impôts à la consommation : MIMSE-CONSO » Document de travail du THEMA. 
ANNEXES 


\section{Annexe 1 : Evolution des taux de TVA}

Avant le $1^{\text {er }}$ juillet 1982 (2), le régime de la TVA comprenait quatre taux.

Le taux réduit (7\%) portait à la fois sur des produits et des prestations de services.

* Les produits soumis au taux réduit étaient :

-ceux destinés à l'alimentation humaine avec quelques exceptions (voir ceux soumis au taux intermédiaire),

-les livres,

-les produits pharmaceutiques,

-les huiles végétales alimentaires.

* Concernant les prestations de services, étaient appliquées un taux réduit sur :

-les transports de voyageurs,

-le logement (hôtels de tourisme et villages de vacances, les locations d'emplacement sur terrains de camping, les gîtes),

-les prestations relatives à la fourniture et à l'évacuation d'eau.

Le taux intermédiaire portait sur des produits et des prestations de services :

* Les produits soumis au taux intermédiaire :

-certains produits alimentaires : la confiserie, certains chocolats et produits contenant du cacao, la margarine, les graisses végétales, les eaux minérales, naturelles et eaux de table ainsi que les boissons alcoolisées et non alcoolisées,

-les produits énergétiques tel que le gaz, l'air comprimé, la vapeur d'eau,

-les produits des exploitations forestières et bois de scierie.

* Concernant les services :

-les ventes à consommer sur place,

-les prestations de services fournies par les personnes répertoriées au répertoire des métiers,

-les soins donnés par les établissements hospitaliers, maisons de repos,

-l'enseignement général, agricole, professionnel.

Le taux normal $(17,6 \%)$ de la TVA était applicable à toutes les opérations imposables pour lesquelles un autre taux n'est pas prévu.

Le taux majoré $(33,3 \%)$ s'appliquait quant à lui sur :

-les véhicules automobiles et motocyclettes,

-les ouvrages composés de pierres, perles et métaux précieux, 
-les appareils d'enregistrement du son ou de l'image,

-les matériels de photographie et de cinématographie,

-les pelleteries provenant de certaines espèces animales et vêtements accessoires,

-les films pornographiques ou d'incitation à la violence.

Entre le $1^{\text {er }}$ juillet 1982 et le 30 décembre 1988 (3), des modifications sont intervenues à savoir l'institution d'un taux super réduit, la fusion du taux normal et du taux intermédiaire ainsi que l'ajout de produits soumis au taux majoré.

Les produits soumis au taux réduit restent les mêmes et seuls les produits destinés à l'alimentation humaine sont soumis au taux super réduit $(5,5 \%)$.

Le caviar, les produits de parfumerie à base d'alcool, les tabacs ont été taxés au taux majoré $(33,3 \%)$

Au $1^{\text {er }}$ janvier 1989 (4), le taux réduit (7\%) a été ramené au niveau du taux super réduit $(5,5 \%)$, ces deux taux étant fusionnés en un taux réduit unique $(5,5 \%)$

Les eaux minérales, naturelles et eaux de table ainsi que les boissons non alcoolisées ont été ramenées au taux réduit .

Enfin, depuis le $1^{\text {er }}$ janvier 1993 (5), le taux majoré n'existe plus et seulement deux taux sont en vigueur et les produits soumis auparavant au taux majoré ont été ramenés au taux normal. 


\section{Annexe 2 : Définition et base d'imposition des boissons alcoolisées}

\section{Encadré 2. Définition et base d'imposition des boissons alcoolisées}

* Les produits alcoolisés au sens de la fiscalité

*. Les vins, cidres, poirés et bières

Sont définies de vins, cidres, poirés et bières ; les boissons à base de raisin, pomme, poire ou houblon, le fruit ou la plante pouvant figurer à tous les niveaux de production.

* Les produits intermédiaires

Ce sont des produits relevant des codes NC 2204, 2205 et 2206 du tarif des douanes, qui contiennent entre $1,2 \%$ et $22 \%$ d'alcool pur, et qui ne sont ni des bières, cidres et poirés, ni du vin. Cette catégorie regroupe typiquement les alcools destinés à l'apéritif.

*. Les « alcools»

Troisième catégorie de produits : ce sont les alcools à proprement parler. Ils désignent les produits qui relèvent des codes NC 2207 et 2208 du code des douanes, qui contiennent plus de $1.2 \%$ d'alcool pur, ainsi que ceux relevant des codes NC 2204, 2205 et 2206 du tarif des douanes, qui contiennent plus de $22 \%$ d'alcool pur (et ne sont donc pas classés dans les produits intermédiaires pour cette raison).

\section{* La base d'imposition des boissons alcoolisées}

Les textes communautaires ont précisé les bases d'imposition à retenir :

*. tarif exprimé en hectolitre de produit pour les vins, les boissons fermentées autres que les vins ainsi que les produits intermédiaires,

*. tarif exprimé en hectolitre de produit par titre alcoométrique acquis pour les bières,

*. tarif exprimé en hectolitre d'alcool pur pour les alcools éthyliques

La taxe CNAM est perçue sur les alcools ayant un titre alcoométrique volumique supérieur à $25 \%$ vol et est redevable à la mise sur le marché des produits. C'est le détaillant qui doit s'acquitter du montant pour ensuite la répercuter sur ses clients. Elle s'élève à 0,13 euros par degré d'alcool.

La taxe CNAM et la taxe Premix poursuivent des finalités de santé publique. 


\section{Annexe 3. Présentation de l'enquête Budget De Famille 2001}

1. Objectifs de l'enquête

L'enquête « Budget de Famille » a pour objectif de décrire avec précision l'ensemble du budget des ménages. La totalité des dépenses des ménages sont enregistrées, avec leur montant et leur nature, ventilée dans une nomenclature d'environ 700 postes budgétaires. Toutes les dépenses sont couvertes y compris celles qui ne relèvent pas de la consommation des biens et services (au sens des Comptes Nationaux) tel que les impôts et taxes ainsi que les consommations ne donnant pas lieu à dépense (autoconsommation alimentaire).

Comme le revenu est le principal déterminant de la consommation, l'enquête Budget enregistre toutes les formes de ressources : revenus imposables ou non, prestations sociales, sommes d'autres ménages, ressources exceptionnelles (loto, héritage, prime de licenciement,...).

2. La description du mode de collecte

Afin de contrôler la saisonnalité, la collecte de l'enquête BDF est répartie sur 12 mois ( 8 vagues de 6 semaines). Elle a débuté le 9 mai 2000 et s'est terminée le 6 mai 2001 avec une interruption de 15 jours en août et de 15 jours en décembre.

L'enquête BDF, comme ses homologues étrangères, utilise deux instruments de collecte:

a) Un questionnaire-ménage informatisé posé par l'enquêteur en face à face

- les caractéristiques socio-démographiques du ménage sont enregistrées

- les dépenses importantes ou régulières : logement et habitation, automobile et biens durables, vêtements de dessus, vacances, garde d'enfants et travaux ménagers, dépenses diverses.

- les ressources perçues par le ménage au cours de la dernière année civile.

- les questions qualitatives illustrant la situation financière du ménage.

Le questionnaire est réparti sur trois visites effectuées par l'enquêteur chez le ménage durant la période de collecte du carnet : 14 jours.

b) Des carnets de compte individuels

Ces carnets sont remplis par les membres du ménage enquêté âgés de plus de 14 ans. Ils enregistrent les dépenses quotidiennes, ainsi que les petites dépenses irrégulières.

Chaque membre âgé de plus de 14 ans du ménage enquêté doit noter sur son carnet toutes les dépenses qu'il effectue durant 14 jours. L'enquêteur distribue les carnets lors de sa première visite, les vérifie 
lors de la seconde, puis les ramasse lors de sa troisième visite. Lors de l'exploitation, les carnets sont chiffrés dans la nomenclature de dépenses de l'enquête. Pour chaque poste de nomenclature, sont retenues finalement :

- les données fournies par le questionnaire, si le poste de dépense est étudié par le questionnaire.

- les données du carnet, si le poste de dépense n'est pas couvert par le questionnaire.

\begin{tabular}{|l|l|l|}
\hline \multicolumn{2}{|l|}{} & $\begin{array}{l}\text { Temps moyen } \\
\text { par visite }\end{array}$ \\
\hline 1ère visite & $\begin{array}{l}\text { Explications + } \\
\text { Questionnaires } \\
\text { Dépôts des carnets }\end{array}$ & 1 heure \\
\hline 2ème visite & Questionnaires & $45 \mathrm{mn}$ \\
\hline & Vérification carnets & $15 \mathrm{mn}$ \\
\hline 3ème visite & Questionnaires & $45 \mathrm{mn}$ \\
\hline & $\begin{array}{l}\text { Vérification carnets + } \\
\text { Ramassage }\end{array}$ & $15 \mathrm{mn}$ \\
\hline
\end{tabular}

Source : BDF 2000-2001 


\section{Annexe 4 Equation de régression du montant total de la fiscalité indirecte}

\begin{tabular}{|l|r|r|r|}
\hline Variable & $\begin{array}{r}\text { Coefficient } \\
\text { estimé }\end{array}$ & $\begin{array}{r}\text { Ecart type } \\
\text { estimé }\end{array}$ & $\begin{array}{r}\text { T de Student } \\
\text { estimé }\end{array}$ \\
\hline constante & 3,09 & 0,11 & 27,5 \\
\hline Log du revenu disponible net & 0,43 & 0,01 & 39,8 \\
\hline Homme seul & $-0,25$ & 0,02 & $-14,1$ \\
Femme seule & $-0,45$ & 0,02 & $-26,5$ \\
Famille monoparentale & $-0,12$ & 0,02 & $-5,1$ \\
Couple + 1 enfant & 0,18 & 0,02 & 10,2 \\
Couple + 2 enfants & 0,19 & 0,02 & 10,0 \\
Couple + 3 enfants & 0,23 & 0,02 & 9,7 \\
Couple sans enfant (référence) & & & \\
\hline Age moins 30 & 0,30 & 0,02 & 17,3 \\
Age 30-40 & 0,14 & 0,02 & 9,1 \\
Age 40-50 & 0,19 & 0,02 & 12,8 \\
Age 50 et plus (référence) & & & \\
\hline Agriculteur & $-0,08$ & 0,02 & $-3,4$ \\
Artisan & 0,07 & 0,02 & 3,1 \\
Cadre & 0,24 & 0,02 & 13,3 \\
Prof intermédiaire & 0,15 & 0,02 & 10,0 \\
Employé & 0,02 & 0,02 & 1,2 \\
Autre & $-0,13$ & 0,02 & $-6,1$ \\
Ouvrier (référence) & & & \\
\hline Bassin Parisien & 0,12 & 0,02 & 7,0 \\
Nord & 0,17 & 0,02 & 7,4 \\
Est & 0,21 & 0,02 & 10,1 \\
Ouest & 0,12 & 0,02 & 6,6 \\
Sud ouest & 0,18 & 0,02 & 9,4 \\
Centre est & 0,17 & 0,02 & 8,7 \\
Méditerranée & 0,11 & 0,02 & 5,8 \\
Paris (référence) & & & \\
\hline
\end{tabular}

\begin{tabular}{|l|r|r|r|r|r|}
\hline \multicolumn{1}{|c|}{ Source } & DL & $\begin{array}{c}\text { Somme } \\
\text { Des carrés }\end{array}$ & $\begin{array}{c}\text { Ecart } \\
\text { Quadratique } \\
\text { moyen }\end{array}$ & $\begin{array}{r}\text { F de Fisher } \\
\text { Snédécor }\end{array}$ & Prob > F \\
\hline Modèle estimé & 23 & 6240393 & 271321 & 439,7 & $<0,0001$ \\
Résidus & 10281 & 6343776 & 617 & & \\
Total & 10304 & 12584169 & & & \\
Erreur quadratique moyenne & 24,8 & & & & \\
Moyenne de la variable expliquée & 7,7 & & & & \\
Coefficient de variation & 324,3 & & & & \\
R2 & 0,5 & & & & \\
R2 ajusté & 0,5 & & & & \\
\hline
\end{tabular}


Annexe 5 Equation de régression du log de montant de TVA à taux réduit (5,5\%)

\begin{tabular}{|l|r|r|r|}
\hline Variable & $\begin{array}{r}\text { Coefficient } \\
\text { estimé }\end{array}$ & $\begin{array}{r}\text { Ecart type } \\
\text { estimé }\end{array}$ & $\begin{array}{r}\text { T de Student } \\
\text { estimé }\end{array}$ \\
\hline constante & 1,38 & 0,12 & 11,1 \\
\hline Log du revenu disponible net & 0,39 & 0,01 & 32,4 \\
\hline Homme seul & $-0,48$ & 0,02 & $-24,9$ \\
Femme seule & $-0,39$ & 0,02 & $-21,2$ \\
Famille monoparentale & $-0,05$ & 0,03 & $-2,2$ \\
Couple + 1 enfant & 0,16 & 0,02 & 8,1 \\
Couple + 2 enfants & 0,23 & 0,02 & 11,2 \\
Couple + 3 enfants & 0,31 & 0,03 & 11,9 \\
Couple sans enfant (référence) & & & \\
\hline Age moins 30 & $-0,32$ & 0,02 & $-16,7$ \\
Age 30-40 & $-0,21$ & 0,02 & $-12,6$ \\
Age 40-50 & $-0,09$ & 0,02 & $-5,2$ \\
Age 50 et plus (référence) & & & \\
\hline Agriculteur & $-0,10$ & 0,03 & $-3,7$ \\
Artisan & 0,03 & 0,02 & 1,2 \\
Cadre & 0,10 & 0,02 & 5,2 \\
Prof intermédiaire & 0,08 & 0,02 & 5,0 \\
Employé & $-0,01$ & 0,02 & $-0,5$ \\
Autre & $-0,05$ & 0,02 & $-2,1$ \\
Ouvrier (référence) & & & \\
\hline Bassin Parisien & 0,06 & 0,02 & 3,1 \\
Nord & 0,15 & 0,03 & 5,8 \\
Est & 0,08 & 0,02 & 3,5 \\
Ouest & 0,03 & 0,02 & 1,6 \\
Sud ouest & 0,09 & 0,02 & 4,0 \\
Centre est & 0,11 & 0,02 & 5,3 \\
Méditerranée & 0,12 & 0,02 & 5,5 \\
Paris (référence) & & & \\
\hline
\end{tabular}

\begin{tabular}{|l|r|r|r|r|r|}
\hline \multicolumn{1}{|c|}{ Source } & DL & $\begin{array}{c}\text { Somme } \\
\text { Des carrés }\end{array}$ & $\begin{array}{c}\text { Ecart } \\
\text { Quadratique } \\
\text { moyen }\end{array}$ & $\begin{array}{c}\text { F de Fisher } \\
\text { Snédécor }\end{array}$ & Prob > F \\
\hline Modèle estimé & 23 & 5603010 & 243609 & 323,23 & $<0,0001$ \\
Résidus & 10281 & 7748459 & 754 & & \\
Total & 10304 & 13351468 & & & \\
Erreur quadratique moyenne & 27,45 & & & & \\
Moyenne de la variable & 5,23 & & & & \\
expliquée & 524,63 & & & \\
Coefficient de variation & 0,42 & & & & \\
R2 & 0,42 & & & & \\
R2 ajusté & & & & & \\
\hline
\end{tabular}


Annexe 6 Equation de régression du log de montant de TVA à taux normal $(19,6 \%)$

\begin{tabular}{|l|r|r|r|}
\hline Variable & $\begin{array}{r}\text { Coefficient } \\
\text { estimé }\end{array}$ & $\begin{array}{r}\text { Ecart type } \\
\text { estimé }\end{array}$ & $\begin{array}{r}\text { T de Student } \\
\text { estimé }\end{array}$ \\
\hline constante & 2,20 & 0,12 & 18,7 \\
\hline Log du revenu disponible net & 0,46 & 0,01 & 40,5 \\
\cline { 2 - 3 } Homme seul & $-0,24$ & 0,02 & $-13,2$ \\
Femme seule & $-0,31$ & 0,02 & $-17,9$ \\
Famille monoparentale & $-0,07$ & 0,02 & $-3,1$ \\
Couple + 1 enfant & 0,18 & 0,02 & 9,8 \\
Couple + 2 enfants & 0,21 & 0,02 & 10,4 \\
Couple + 3 enfants & 0,25 & 0,02 & 10,2 \\
Couple sans enfant (référence) & & & \\
\hline Age moins 30 & 0,32 & 0,02 & 17,4 \\
Age 30-40 & 0,16 & 0,02 & 9,9 \\
Age 40-50 & 0,19 & 0,02 & 12,1 \\
Age 50 et plus (référence) & & & \\
\hline Agriculteur & $-0,09$ & 0,03 & $-3,5$ \\
Artisan & 0,12 & 0,02 & 5,5 \\
Cadre & 0,33 & 0,02 & 17,6 \\
Prof intermédiaire & 0,20 & 0,02 & 12,5 \\
Employé & 0,05 & 0,02 & 3,1 \\
Autre & $-0,05$ & 0,02 & $-2,3$ \\
Ouvrier (référence) & & & \\
\hline Bassin Parisien & 0,06 & 0,02 & 3,1 \\
Nord & 0,12 & 0,02 & 4,8 \\
Est & 0,18 & 0,02 & 8,3 \\
Ouest & 0,07 & 0,02 & 3,6 \\
Sud ouest & 0,12 & 0,02 & 5,7 \\
Centre est & 0,12 & 0,02 & 5,8 \\
Méditerranée & 0,05 & 0,02 & 2,5 \\
Paris (référence) & & & \\
\hline
\end{tabular}

\begin{tabular}{|l|c|c|l|l|l|}
\hline \multicolumn{1}{|c|}{ Source } & DL & $\begin{array}{c}\text { Somme } \\
\text { Des carrés }\end{array}$ & $\begin{array}{c}\text { Ecart } \\
\text { Quadratique } \\
\text { moyen }\end{array}$ & $\begin{array}{c}\text { F de Fisher } \\
\text { Snédécor }\end{array}$ & Prob > F \\
\hline Modèle estimé & 23 & 6239616 & 271288 & 400 & $<0,0001$ \\
Résidus & 10281 & 6974850 & 678 & & \\
Total & 10304 & 13214465 & & & \\
Erreur quadratique moyenne & 26,0 & & & & \\
Moyenne de la variable expliquée & 7,1 & & & & \\
Coefficient de variation & 0,5 & & & & \\
R2 & 0,5 & & & & \\
\hline R2 ajusté & & & & & \\
\hline
\end{tabular}


Annexe 7 Equation de régression du montant total de TVA

\begin{tabular}{|l|r|r|r|}
\hline Variable & $\begin{array}{r}\text { Coefficient } \\
\text { estimé }\end{array}$ & $\begin{array}{c}\text { Ecart type } \\
\text { estimé }\end{array}$ & $\begin{array}{c}\text { T de Student } \\
\text { estimé }\end{array}$ \\
\hline Constante & 2,65 & 0,11 & 25,0 \\
\hline Log du revenu disponible net & 0,44 & 0,01 & 42,9 \\
\hline Homme seul & $-0,27$ & 0,02 & $-16,4$ \\
Femme seule & $-0,33$ & 0,02 & $-20,7$ \\
Famille monoparentale & $-0,08$ & 0,02 & $-3,6$ \\
Couple + 1 enfant & 0,17 & 0,02 & 10,2 \\
Couple + 2 enfants & 0,20 & 0,02 & 11,3 \\
Couple + 3 enfants & 0,25 & 0,02 & 11,3 \\
Couple sans enfant (référence) & & & \\
\hline Age moins 30 & 0,23 & 0,02 & 14,1 \\
Age 30-40 & 0,10 & 0,01 & 7,3 \\
Age 40-50 & 0,15 & 0,01 & 10,6 \\
Age 50 et plus (référence) & & & \\
\hline Agriculteur & $-0,09$ & 0,02 & $-3,7$ \\
Artisan & 0,11 & 0,02 & 5,4 \\
Cadre & 0,29 & 0,02 & 17,5 \\
Prof intermédiaire & 0,18 & 0,01 & 12,4 \\
Employé & 0,04 & 0,02 & 2,7 \\
Autre & $-0,05$ & 0,02 & $-2,6$ \\
Ouvrier (référence) & & & \\
\hline Bassin Parisien & 0,05 & 0,02 & 3,0 \\
Nord & 0,11 & 0,02 & 5,1 \\
Est & 0,16 & 0,02 & 8,0 \\
Ouest & 0,06 & 0,02 & 3,2 \\
Sud ouest & 0,10 & 0,02 & 5,6 \\
Centre est & 0,11 & 0,02 & 6,0 \\
Méditerranée & 0,06 & 0,02 & 3,1 \\
Paris (référence) & & & \\
\hline
\end{tabular}

\begin{tabular}{|l|r|r|r|r|r|}
\hline \multicolumn{1}{|c|}{ Source } & DL & $\begin{array}{c}\text { Somme } \\
\text { Des carrés }\end{array}$ & $\begin{array}{c}\text { Ecart } \\
\text { Quadratique } \\
\text { moyen }\end{array}$ & $\begin{array}{c}\text { F de Fisher } \\
\text { Snédécor }\end{array}$ & Prob > F \\
\hline Modèle estimé & 23 & 5718410 & 248627 & $453,1<0,0001$ \\
Résidus & 10281 & 5641483 & 549 & & \\
Total & 10304 & 11359893 & & & \\
Erreur quadratique moyenne & 23,42 & & & & \\
Moyenne de la variable expliquée & 7,27 & & & & \\
Coefficient de variation & 322,14 & & & & \\
R2 & 0,50 & & & & \\
R2 ajusté & 0,50 & & & & \\
\hline
\end{tabular}


Annexe 8 Equation de sélection et de régression pour les taxes sur l’alcool

\section{Equation de sélection pour les taxes sur l'alcool}

\begin{tabular}{|c|c|c|c|}
\hline Variable & $\begin{array}{l}\text { Coefficient } \\
\text { estimé }\end{array}$ & $\begin{array}{c}\text { Ecart type } \\
\text { estimé }\end{array}$ & Chi2 \\
\hline Constante & 0,50 & 0,05 & 10,9 \\
\hline Sans diplôme & $-0,06$ & 0,03 & $-1,9$ \\
\hline Bac (gén+tech) & $-0,01$ & 0,04 & $-0,2$ \\
\hline $\mathrm{Bac}+2 \mathrm{et}+$ & $-0,07$ & 0,04 & $-2,0$ \\
\hline CAP- BEP Bepc (référence) & & & \\
\hline Commune rurale & 0,07 & 0,04 & 1,6 \\
\hline Moins 20000 habitants & 0,12 & 0,05 & 2,5 \\
\hline Entre 20et $100000 \mathrm{hab}$ & 0,03 & 0,05 & 0,6 \\
\hline Plus de 100000 hab & 0,10 & 0,04 & 2,4 \\
\hline Aglom parisienne (référence) & & & \\
\hline Homme seul & $-0,33$ & 0,04 & $-7,7$ \\
\hline Femme seule & $-0,62$ & 0,04 & $-15,9$ \\
\hline Famille monoparentale & $-0,47$ & 0,05 & $-8,9$ \\
\hline Couple +1 enfant & $-0,05$ & 0,04 & $-1,2$ \\
\hline Couple +2 enfants & $-0,07$ & 0,04 & $-1,6$ \\
\hline Couple +3 enfants & $-0,15$ & 0,05 & $-2,8$ \\
\hline Couple sans enfant (référence) & & & \\
\hline
\end{tabular}

\section{Equation de régression du log de montant de taxes sur l'alcool}

\begin{tabular}{|c|c|c|c|}
\hline Variable & $\begin{array}{l}\text { Coefficient } \\
\text { estimé }\end{array}$ & $\begin{array}{l}\text { Ecart type } \\
\text { estimé }\end{array}$ & $\begin{array}{l}\text { T de Student } \\
\text { estimé }\end{array}$ \\
\hline constante & 2,28 & 1,10 & 2,1 \\
\hline Log du revenu disponible net & 0,16 & 0,07 & 2,5 \\
\hline Homme seul & 0,41 & 0,34 & 1,2 \\
\hline Femme seule & 0,23 & 0,63 & 0,4 \\
\hline Famille monoparentale & 0,47 & 0,48 & 1,0 \\
\hline Couple +1 enfant & 0,15 & 0,13 & 1,2 \\
\hline Couple +2 enfants & 0,14 & 0,14 & 1,0 \\
\hline Couple +3 enfants & 0,25 & 0,21 & 1,2 \\
\hline \multicolumn{4}{|l|}{ Couple sans enfant (référence) } \\
\hline Age moins 30 & $-0,28$ & 0,14 & $-2,0$ \\
\hline Age $30-40$ & $-0,28$ & 0,09 & $-3,0$ \\
\hline Age 40-50 & $-0,02$ & 0,08 & $-0,2$ \\
\hline \multicolumn{4}{|l|}{ Age 50 et plus (référence) } \\
\hline Agriculteur & $-0,16$ & 0,16 & $-1,0$ \\
\hline Artisan & $-0,12$ & 0,12 & $-1,1$ \\
\hline Cadre & $-0,15$ & 0,10 & $-1,4$ \\
\hline Prof intermédiaire & $-0,08$ & 0,09 & $-0,9$ \\
\hline Employé & $-0,12$ & 0,10 & $-1,2$ \\
\hline Autre & $-0,02$ & 0,15 & $-0,2$ \\
\hline \multicolumn{4}{|l|}{ Ouvrier (référence) } \\
\hline Bassin Parisien & 0,05 & 0,14 & 0,4 \\
\hline Nord & 0,53 & 0,16 & 3,2 \\
\hline Est & $-0,03$ & 0,15 & $-0,2$ \\
\hline Ouest & $-0,06$ & 0,14 & $-0,4$ \\
\hline Sud ouest & $-0,37$ & 0,15 & $-2,4$ \\
\hline Centre est & $-0,29$ & 0,15 & $-2,0$ \\
\hline Méditerranée & $-0,35$ & 0,15 & $-2,3$ \\
\hline Ratio mills & $-1,96$ & 0,67 & $-2,9$ \\
\hline \multicolumn{4}{|l|}{ Paris (référence) } \\
\hline rho & $-0,95$ & & \\
\hline sigma & 2,55 & & \\
\hline Mills (lambda) & $-2,41$ & 1,66 & \\
\hline
\end{tabular}


Annexe 9 Equation de sélection et régression pour les taxes sur le tabac

\section{Equation de sélection pour les taxes sur le tabac}

\begin{tabular}{|l|r|r|r|}
\hline Variable & \multicolumn{1}{|c|}{$\begin{array}{c}\text { Coefficient } \\
\text { estimé }\end{array}$} & \multicolumn{2}{|c|}{$\begin{array}{c}\text { Ecart type } \\
\text { estimé }\end{array}$} \\
\hline Constante & $-0,56$ & 0,05 & $-11,8$ \\
\hline Sans diplôme & $-0,30$ & 0,03 & $-9,2$ \\
Bac (gén+tech) & $-0,06$ & 0,04 & $-1,3$ \\
Bac + 2 et + & $-0,15$ & 0,04 & $-4,0$ \\
CAP- BEP Bepc (réf) & $-0,09$ & 0,05 & $-1,9$ \\
\hline Commune rurale & $-0,04$ & 0,05 & $-0,9$ \\
Moins 20000 habitants & 0,07 & 0,05 & 1,4 \\
Entre 20et 100000 hab & 0,05 & 0,04 & 1,2 \\
Plus de 100000 hab & & & 6,2 \\
Aglom parisienne (réf) & 0,28 & 0,04 & $-4,5$ \\
\hline Homme seul & $-0,20$ & 0,04 & 10,9 \\
Femme seule & 0,57 & 0,05 & 12,9 \\
Famille monoparentale & 0,55 & 0,04 & 12,4 \\
Couple + 1 enfant & 0,51 & 0,04 & 10,1 \\
Couple + 2 enfants & 0,53 & 0,05 & \\
Couple + 3 enfants & & & \\
Couple sans enfant (réf) & & & \\
\hline
\end{tabular}

\section{Equation de régression du log de montant de taxes sur le tabac}

\begin{tabular}{|c|c|c|c|}
\hline Variable & $\begin{array}{c}\text { Coefficient } \\
\text { estimé }\end{array}$ & $\begin{array}{l}\text { Ecart type } \\
\text { estimé }\end{array}$ & $\begin{array}{l}\text { T de Student } \\
\text { estimé }\end{array}$ \\
\hline constante & 5,41 & 0,87 & 6,2 \\
\hline Log du revenu disponible net & 0,02 & 0,07 & 0,2 \\
\hline Homme seul & 0,03 & 0,14 & 0,2 \\
\hline Femme seule & $-0,09$ & 0,17 & $-0,5$ \\
\hline Famille monoparentale & $-0,11$ & 0,18 & $-0,6$ \\
\hline Couple +1 enfant & 0,12 & 0,16 & 0,7 \\
\hline Couple +2 enfants & 0,03 & 0,15 & 0,2 \\
\hline Couple +3 enfants & 0,16 & 0,17 & 0,9 \\
\hline \multicolumn{4}{|l|}{ Couple sans enfant (référence) } \\
\hline Age moins 30 & 0,11 & 0,10 & 1,0 \\
\hline Age $30-40$ & 0,07 & 0,08 & 0,9 \\
\hline Age 40-50 & 0,10 & 0,08 & 1,2 \\
\hline \multicolumn{4}{|l|}{ Age 50 et plus (référence) } \\
\hline Agriculteur & $-0,42$ & 0,28 & $-1,5$ \\
\hline Artisan & $-0,06$ & 0,14 & $-0,4$ \\
\hline Cadre & $-0,17$ & 0,10 & $-1,7$ \\
\hline Prof intermédiaire & $-0,09$ & 0,08 & $-1,1$ \\
\hline Employé & $-0,07$ & 0,09 & $-0,8$ \\
\hline Autre & $-0,13$ & 0,12 & $-1,0$ \\
\hline \multicolumn{4}{|l|}{ Ouvrier (référence) } \\
\hline Bassin Parisien & 0,02 & 0,10 & 0,2 \\
\hline Nord & $-0,07$ & 0,13 & $-0,5$ \\
\hline Est & $-0,06$ & 0,12 & $-0,5$ \\
\hline Ouest & $-0,05$ & 0,11 & $-0,5$ \\
\hline Sud ouest & $-0,11$ & 0,12 & $-0,9$ \\
\hline Centre est & $-0,04$ & 0,12 & $-0,3$ \\
\hline Méditerranée & $-0,06$ & 0,12 & $-0,5$ \\
\hline \multicolumn{4}{|l|}{ Paris (référence) } \\
\hline rho & $-0,25$ & & \\
\hline sigma & 0,99 & & \\
\hline Mills ( lambda) & $-0,25$ & 0,35 & \\
\hline
\end{tabular}


Annexe 10 Equation de sélection et régression pour les taxes sur les jeux de hasard

\section{Equation de sélection pour les taxes sur les jeux de hasard}

\begin{tabular}{|l|r|r|r|}
\hline Variable & \multicolumn{1}{|c|}{$\begin{array}{c}\text { Coefficient } \\
\text { estimé }\end{array}$} & \multicolumn{2}{|c|}{$\begin{array}{c}\text { Ecart type } \\
\text { estimé }\end{array}$} \\
\hline Constante & $-0,82$ & 0,05 & $-15,8$ \\
\hline Sans diplôme & $-0,10$ & 0,03 & $-3,0$ \\
Bac (gén+tech) & $-0,23$ & 0,05 & $-4,7$ \\
Bac + 2 et + & $-0,34$ & 0,04 & $-8,4$ \\
CAP- BEP Bepc (référence) & & & 0,7 \\
\hline Commune rurale & 0,04 & 0,05 & 4,0 \\
Moins 20000 habitants & 0,21 & 0,05 & 2,4 \\
Entre 20et 100000 hab & 0,13 & 0,06 & 4,9 \\
Plus de 100000 hab & 0,24 & 0,05 & $-3,1$ \\
Aglom parisienne (référence) & & & $-6,7$ \\
\hline Homme seul & $-0,16$ & 0,05 & $-1,8$ \\
Femme seule & $-0,31$ & 0,05 & 5,0 \\
Famille monoparentale & $-0,11$ & 0,06 & 6,1 \\
Couple + 1 enfant & 0,23 & 0,05 & 4,8 \\
Couple + 2 enfants & 0,27 & 0,04 & \\
Couple + 3 enfants & 0,26 & 0,05 & \\
Couple sans enfant (référence) & & & \\
\hline
\end{tabular}

\section{Equation de régression du log de montant des taxes sur les jeux de hasard}

\begin{tabular}{|l|r|r|r|}
\hline Variable & $\begin{array}{r}\text { Coefficient } \\
\text { estimé }\end{array}$ & $\begin{array}{c}\text { Ecart type } \\
\text { estimé }\end{array}$ & $\begin{array}{c}\text { T de Student } \\
\text { estimé }\end{array}$ \\
\hline constante & 2,76 & 1,82 & 1,5 \\
\hline Log du revenu disponible net & 0,13 & 0,17 & 0,7 \\
\hline Homme seul & 0,25 & 0,23 & 1,1 \\
Femme seule & $-0,28$ & 0,27 & $-1,0$ \\
Famille monoparentale & 0,03 & 0,25 & 0,1 \\
Couple + 1 enfant & $-0,04$ & 0,17 & $-0,2$ \\
Couple + 2 enfants & $-0,06$ & 0,18 & $-0,3$ \\
Couple + 3 enfants & 0,02 & 0,21 & 0,1 \\
Couple sans enfant (référence) & & & \\
\hline Age moins 30 & $-0,36$ & 0,20 & $-1,8$ \\
Age 30-40 & $-0,30$ & 0,14 & $-2,1$ \\
Age 40-50 & $-0,20$ & 0,13 & $-1,6$ \\
Age 50 et plus (référence) & & & $-0,3$ \\
\hline Agriculteur & $-0,11$ & 0,38 & 0,1 \\
Artisan & 0,02 & 0,26 & $-0,1$ \\
Cadre & $-0,03$ & 0,21 & $-0,8$ \\
Prof intermédiaire & $-0,11$ & 0,13 & $-0,9$ \\
Employé & $-0,13$ & 0,14 & $-0,4$ \\
Autre & $-0,11$ & 0,29 & $-0,2$ \\
Ouvrier (référence) & & & $-0,2$ \\
\hline Bassin Parisien & $-0,04$ & 0,20 & $-0,1$ \\
Nord & $-0,05$ & 0,21 & $-0,3$ \\
Est & $-0,02$ & 0,22 & 0,7 \\
Ouest & $-0,06$ & 0,21 & $-0,3$ \\
Sud ouest & 0,16 & 0,23 & 0,9 \\
Centre est & $-0,06$ & 0,22 & \\
Méditerranée & 0,19 & 0,21 & \\
Région Parisien (référence) & $-0,11$ & & \\
\hline ho & 1,06 & & \\
sigma & $-0,12$ & 0,50 & \\
Mills ( lambda) & & & \\
\hline
\end{tabular}


Annexe 11 Equation de sélection et régression pour les taxes sur les carburants (TIPP)

\section{Equation de sélection pour les taxes sur les carburants (TIPP)}

\begin{tabular}{|c|c|c|c|}
\hline Variable & $\begin{array}{l}\text { Coefficient } \\
\text { estimé }\end{array}$ & $\begin{array}{l}\text { Ecart type } \\
\text { estimé }\end{array}$ & Chi2 \\
\hline Constante & 0,23 & 0,05 & 23,4 \\
\hline Sans diplôme & $-0,42$ & 0,03 & $-12,2$ \\
\hline Bac (gén+tech) & 0,02 & 0,05 & 0,4 \\
\hline $\begin{array}{l}\text { Bac }+2 \text { et }+ \\
\text { CAP- BEP Bepc (référence) }\end{array}$ & 0,13 & 0,04 & 3,1 \\
\hline Commune rurale & 0,92 & 0,05 & 19,3 \\
\hline Moins 20000 habitants & 0,70 & 0,05 & 14,1 \\
\hline Entre 20et 100000 hab & 0,66 & 0,05 & 12,6 \\
\hline $\begin{array}{l}\text { Plus de } 100000 \text { hab } \\
\text { Aglom parisienne (référence) }\end{array}$ & 0,48 & 0,04 & 11,1 \\
\hline Homme seul & $-0,34$ & 0,05 & $-7,6$ \\
\hline Femme seule & $-0,89$ & 0,04 & $-22,0$ \\
\hline Famille monoparentale & $-0,32$ & 0,05 & $-5,9$ \\
\hline Couple +1 enfant & 0,33 & 0,05 & 6,5 \\
\hline Couple +2 enfants & 0,32 & 0,05 & 6,6 \\
\hline Couple +3 enfants & 0,39 & 0,06 & 6,2 \\
\hline Couple sans enfant (référence) & & & \\
\hline
\end{tabular}

Equation de régression du log de montant des taxes sur les carburants (TIPP)

\begin{tabular}{|c|c|c|c|}
\hline & Coefficient & Ecart type & T de Student \\
\hline Variable & estimé & estimé & estimé \\
\hline constante & 3,50 & 0,27 & 13,1 \\
\hline Log du revenu disponible net & 0,31 & 0,02 & 12,8 \\
\hline Homme seul & 0,15 & 0,05 & 3,1 \\
\hline Femme seule & 0,06 & 0,08 & 0,8 \\
\hline Famille monoparentale & 0,05 & 0,06 & 0,7 \\
\hline Couple +1 enfant & 0,07 & 0,04 & 1,8 \\
\hline Couple +2 enfants & 0,06 & 0,04 & 1,4 \\
\hline Couple +3 enfants & 0,05 & 0,05 & 0,9 \\
\hline \multicolumn{4}{|l|}{ Couple sans enfant (référence) } \\
\hline Age moins 30 & 0,18 & 0,04 & 4,6 \\
\hline Age 30-40 & 0,01 & 0,03 & 0,4 \\
\hline Age 40-50 & 0,09 & 0,03 & 3,0 \\
\hline \multicolumn{4}{|l|}{ Age 50 et plus (référence) } \\
\hline Agriculteur & $-0,24$ & 0,05 & $-4,7$ \\
\hline Artisan & 0,01 & 0,05 & 0,2 \\
\hline Cadre & 0,11 & 0,04 & 3,1 \\
\hline Prof intermédiaire & 0,09 & 0,03 & 2,8 \\
\hline Employé & 0,05 & 0,04 & 1,3 \\
\hline Autre & $-0,07$ & 0,07 & $-1,1$ \\
\hline \multicolumn{4}{|l|}{ Ouvrier (référence) } \\
\hline Bassin Parisien & $-0,14$ & 0,05 & $-2,6$ \\
\hline Nord & $-0,09$ & 0,06 & $-1,5$ \\
\hline Est & $-0,05$ & 0,06 & $-0,9$ \\
\hline Ouest & $-0,15$ & 0,05 & $-2,8$ \\
\hline Sud ouest & $-0,13$ & 0,06 & $-2,2$ \\
\hline Centre est & $-0,08$ & 0,06 & $-1,4$ \\
\hline Méditerranée & $-0,07$ & 0,06 & $-1,3$ \\
\hline \multicolumn{4}{|l|}{ Paris (référence) } \\
\hline rho & $-0,80$ & & \\
\hline sigma & 0,91 & & \\
\hline Mills ( lambda) & $-0,72$ & 0,12 & \\
\hline
\end{tabular}

